\title{
EVALUACIÓN PALEOSÍSMICA DEL SEGMENTO SAN FELIPE DE LA FALLA DE BOCONÓ (VENEZUELA NOROCCIDENTAL): ¿RESPONSABLE DEL TERREMOTO DEL 26 DE MARZO DE 1812?
}

Franck A. Audemard M. ${ }^{1,2}$

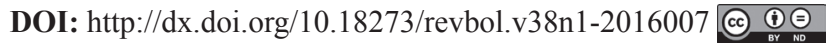

Forma de citar: Audemard, F.A. 2016. Evaluación paleosísmica del segmento San Felipe de la Falla de Boconó (Venezuela noroccidental): ¿responsable del terremoto del 26 de marzo de 1812?. Boletín de Geología, 38 (1): 125-149.

\section{RESUMEN}

La Falla de Boconó (FB), estructura mayor de la frontera transpresiva entre las placas Caribe y Suramérica, presenta claras evidencias geomórficas diagnósticas de transcurrencia dextral cuaternaria en su sector más septentrional (Boc-e), ubicado a lo largo del margen noroeste de la depresión del río Yaracuy. Dos excavaciones paleosísmicas, ejecutadas en las localidades de Yaritagua (El Salto) y Quigua, en proximidad a Barquisimeto y San Felipe, respectivamente, en el año 2005, no sólo confirmaron su actividad tectónica durante el Holoceno, sino que determinaron que la FB es responsable igualmente del sismo destructor del 26 de marzo de 1812, el cual parece componerse de dos subeventos con epicentros macrosísmicos: uno en el mar al ONO de Caracas; y otro entre Barquisimeto y San Felipe, al pie de la sierra de Aroa, en el sector de la FB bajo estudio. Una grieta abierta rellena en El Salto, cuya edad resulta más joven que el año 1770 DC, es la evidencia de este sismo. Por su parte, FB en Quigua recurre con sismos $\mathrm{M}_{\mathrm{w}} \geq 7,0$ cada 2.000-3.000 años, desconociéndose si ésta es toda su actividad sísmica y haciendo presumir que la deformación aquí debe estar distribuida en una amplia zona de deformación activa.

Palabras clave: Falla de Boconó, paleosismicidad, sismicidad histórica, sismo de Caracas de 1812, lluvia cincuentenaria, valle del Yaracuy.

\section{PALEOSEISMIC ASSESSMENT OF THE SAN FELIPE SEGMENT OF THE BOCONÓ FAULT (NORTHWESTERN VENEZUELA): RESPONSIBLE FOR THE MARCH 26TH, 1812 EARTHQUAKE?}

\begin{abstract}
The Boconó Fault (BF), a major tectonic feature of the current transpressive plate boundary zone between the Caribbean and South America, exhibits a large number of Quaternary strike-slip landforms on its northernmost sector (Boc-e), along the NW edge of the Yaracuy tectonic depression. Two paleoseismic trenches, excavated in the vicinity of Yaritagua (El Salto) and Quigua, close to the cities of Barquisimeto and San Felipe respectively, in 2005, not only confirmed the Holocene activity of the BF in this fault portion, but also allowed the ascription to BF of the historical destructive earthquake of March 26th, 1812, which appears to have had two intensity centers: one offshore meizoseismal area WNW of Caracas; and the other one between Barquisimeto and San Felipe, at the foothills of the Aroa range, in the fault portion under study. An organic-rich filled open crack at El Salto trench, whose ${ }^{14} \mathrm{C}$ age is younger than $1770 \mathrm{AD}$, is the evidence to this earthquake. On the other hand, $\mathrm{M}_{\mathrm{w}}$ $\geq 7.0$ earthquakes on the BF at Quigua recurs every 2000-3000 years. It is thought that present-day deformation along and across Boc-e may be accommodated by a wider zone.
\end{abstract}

Keywords: Boconó fault, paleoseismicity, historical seismicity, Caracas 1812 earthquake, fifty-year rain, Yaracuy valley.

${ }^{1}$ Fundación Venezolana de Investigaciones Sismológicas (FUNVISIS). Departamento de Ciencias de la Tierra, Apartado Postal 76880, Caracas 1070-A, Venezuela, faudemard@funvisis.gob.ve.

${ }^{2}$ Universidad Central de Venezuela, Fac. Ingeniería, Escuela de Geología, Minas y Geofísica, Ciudad Universitaria, Los Chaguaramos, Caracas, franck.audemard@ing.ucv.ve 


\section{INTRODUCCION}

El occidente venezolano ha sido el seno de grandes terremotos destructores en su pasado histórico (e.g., Centeno Graü, 1940; Fiedler, 1961; Grases et al., 1999). Al respecto, Rod (1956a) adscribe la gran mayoría de los más importantes sismos a la Falla de Boconó (FB). Igualmente, Cluff and Hansen (1969) le adjudican tanto los principales sismos históricos como instrumentales (contemporáneos). Particularmente, estos autores responsabilizan a la FB por los sismos de 1610, 1812, 1894, 1932 y 1950. Adicionalmente a los eventos de 1610, 1812 y 1894, Aggarwal (1983) -posteriormente citado textualmente por McCann and Pennington (1990) y Suárez and Nábelek (1990)también le asignan los sismos de 1644 y 1875 . Por su parte, Grases (1990), de igual manera que lo hicieran Cluff and Hansen (1969) con anterioridad, indica una probable asociación del sismo de El Tocuyo de 1950 con la FB. En contraposición, Choy (1998) y Audemard et al. (1999b) proponen que otras fallas cercanas pudiesen potencialmente ser también la fuente de ese evento, apoyándose en la complejidad estructural que presenta la región epicentral ubicada al oeste de la FB. Por su parte, Singer and Beltrán (1996) asocian los sismos de Pamplona de 1644 y de Cúcuta de 1875 al indentor de Pamplona (referirse a Boinet, 1985, para la definición de esta estructura; FIGURA 1), y particularmente el de 1875 lo adscriben a la falla de Aguas Calientes, la cual es una falla paralela y al norte de la FB, en proximidad a la frontera colombo-venezolana (FIGURA 2).

De lo antes expuesto, es evidente que las asociaciones sismotectónicas para los terremotos históricos y contemporáneos más importantes ocurridos en los Andes de Mérida varían de autor a autor y de hecho son pocos los sismos antes indicados que tengan claras asociaciones sismotectónicas con la FB, si se exceptúan los trabajos paleosísmicos del autor (Audemard 1997; 1998; 2010; Audemard et al., 2008).

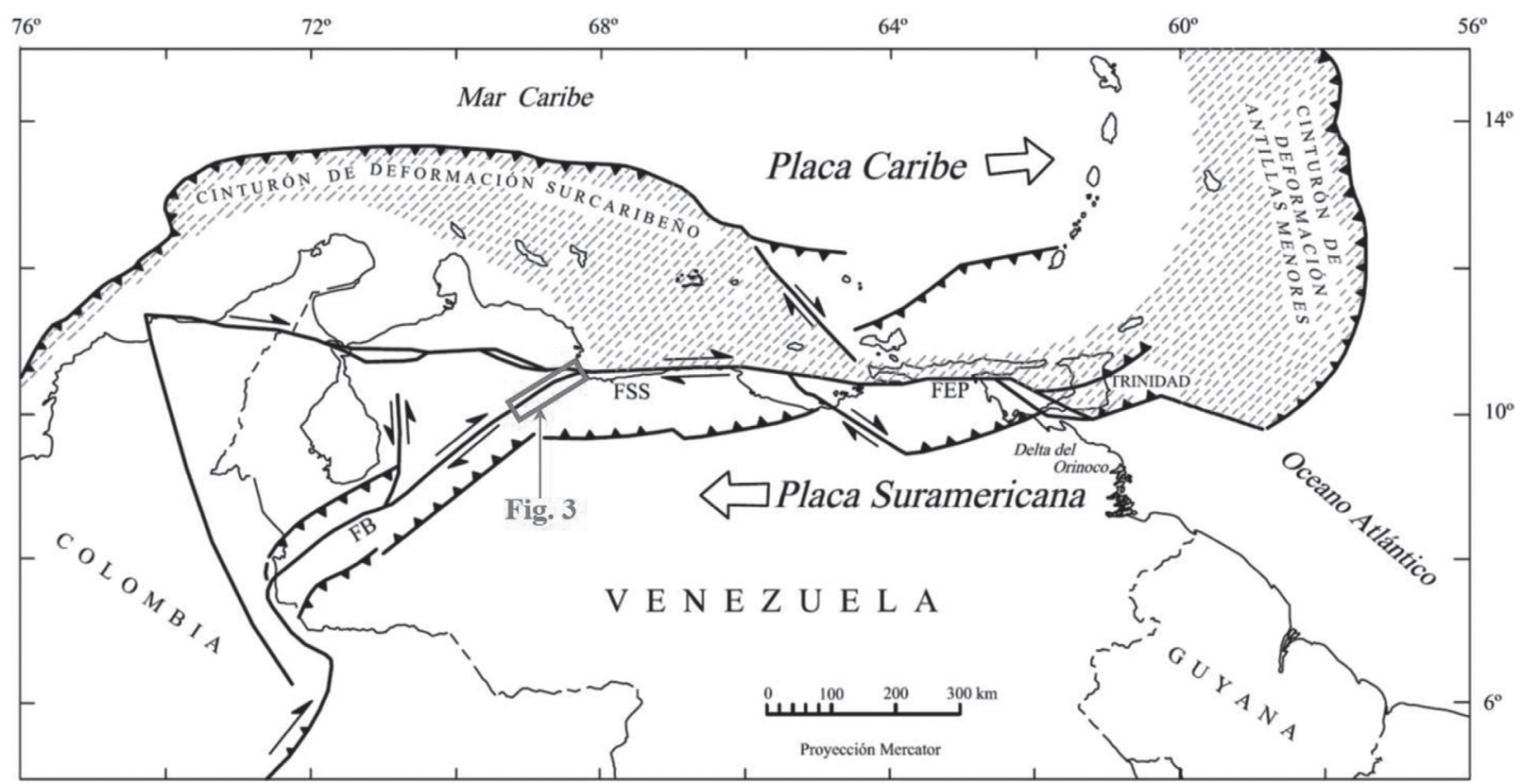

FIGURA 1. Marco geodinámico simplificado del límite sur de la Placa Caribe (modificado de Audemard et al., 2000). Se señala la ubicación relativa de las FIGURAS 2 y 3. Leyenda: FB Falla de Boconó; FSS, Falla de San Sebastián; FEP, Falla El Pilar

A fin de reducir las ambigüedades aún existentes en cuanto a la relación causa-efecto entre la FB y estos sismos destructores históricos, la Fundación Venezolana de Investigaciones Sismológicas -FUNVISIS- ha desarrollado un programa de excavación de trincheras de evaluación paleosísmica a lo largo de los últimos 30 años, iniciados en proximidad a la frontera colombovenezolana, en el estado Táchira en el año 1986 (Audemard and Soulas, 1995; Audemard, 1997; 1998;
Audemard et al., 1999a; 2008; Audemard, 2003a; 2005). Es a partir de estos primeros estudios que los sismos de Mocotíes de 1610 y el Gran Terremoto de los Andes Venezolanos de 1894 son finalmente asociados por vía geológica (paleosísmica) con el segmento más meridional de la FB en los Andes de Mérida (Audemard, 1997; 1998; FIGURA 2). En tiempos más recientes, un fuerte apoyo financiero del gobierno nacional en el presente siglo por intermedio de un proyecto FONACIT 
ha permitido proseguir con este programa (Bueno et al., 2005a y b; Ollarves et al., 2005, Alvarado et al., 2007; 2008; Alvarado, 2008; Audemard, 2008; Audemard et al., 2008; Alvarado et al., 2009; Audemard, 2009c;
Bueno, 2009; Audemard, 2010; 2014), con la ejecución de nuevas trincheras en las diferentes secciones o segmentos de la FB propuestos por Audemard et al. (2000).

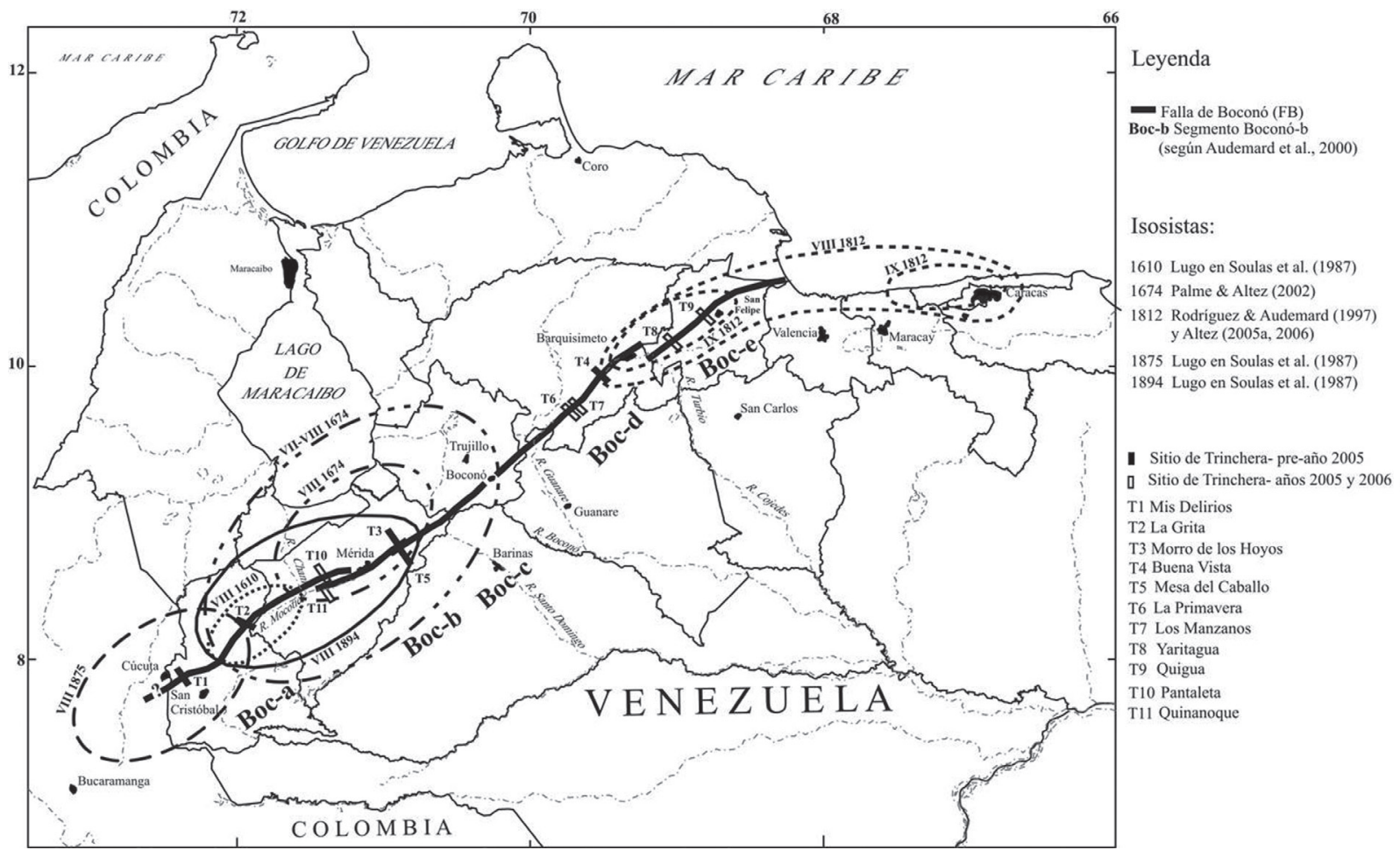

FIGURA 2. Ubicación relativa de las excavaciones paleosísmicas realizadas entre 1986 y 2006 sobre la Falla de Boconó (según Audemard, 2008; 2010). Se muestra igualmente la segmentación de esta falla mayor propuesta por Audemard et al. (2000), así como las isosistas de intensidad VIII o superior de los principales sismos históricos discutidos en este trabajo. Se muestra tal isosista porque ésta es representativa de la longitud de ruptura del sismo responsable. El sismo de 1812 particularmente muestra que se compone de dos subeventos, uno con foco a lo largo del segmento Boc-e de la FB en el valle del Yaracuy, mientras el segundo ocurre costa afuera a lo largo de la costa centro-norte de Venezuela.

Con el mismo interés de reducir los niveles de incertidumbre en la relación causal de los grandes sismos históricos del occidente venezolano, varios colegas especialistas de la sismología histórica han centrado esfuerzos en el análisis de estos eventos, pero a partir de las intensidades macrosísmicas (evaluación macrosísmica). Particular mención ameritan los trabajos de: Rengifo y Laffaille $(1998 ; 2000)$ en lo atinente al Gran Terremoto de los Andes Venezolanos de 1894; Palme y Altez (2002) sobre los sismos de 1673-1674 y; Rodríguez y Audemard (1997), Altez (2005a) y Choy et al. (2010) en referencia al sismo del 26 de marzo de 1812. Los estudios de Palme y Altez (2002) y Choy et al. (2010) que han usado, adaptado y mejorado el método desarrollado por Bakun and Wentworth (1997), han arrojado excelentes resultados en la determinación de las magnitudes y el refinamiento de los epicentros macrosísmicos de los sismos de 1673-1674 y 1812, respectivamente. Particular interés para este estudio reside en la evaluación del sismo de 1812.

De manera independiente, pero complementaria, Audemard (2002), quien aplicando el concepto de silencio sísmico (seismic gap), propone la ubicación y extensión lateral de las rupturas asociadas a los sismos de 1812 (a lo largo del valle del Yaracuy y a lo largo de la costa norcentral en su sector occidental) y 1894 (a lo largo del sector venezolano más meridional) a lo largo de la FB, exclusivamente a partir de la simple lectura visual de la sismicidad instrumental nacional registrada y catalogada por FUNVISIS durante el período 1910-1999.

El objetivo del presente artículo persigue evaluar la asociación sismotectónica del sismo del 26 de marzo de 1812 con el segmento Boconó-e (Boc-e) de la FB (tal como seccionada por Audemard et al., 2000; Audemard, 
2014) a partir de la comparación de las evaluaciones macrosísmicas existentes (Rodríguez y Audemard, 1997; Altez, 2005a y Choy et al., 2010), la posible determinación de su ruptura con base en la aplicación del concepto de silencio sísmico (Audemard, 2002) y los resultados arrojados por dos excavaciones paleosísmicas ejecutadas en el segmento Boc-e (Ollarves et al., 2005 y este estudio). A tal fin, debe tenerse presente que sólo la ruptura cosísmica de los más grandes terremotos puede alcanzar la superficie; es decir, que sólo los sismos históricos o pre-históricos de mayor tamaño, magnitud próxima o superior a $\mathrm{M}_{\mathrm{s}} 7,0$, podrán aquí ser tratados por ser limitaciones propias e intrínsecas de los métodos de evaluación paleosísmica por trincheras (referirse a Audemard, 2003a; 2005; Audemard and Michetti, 2011, para mayor detalle). El sismo de 1812 parece satisfacer esta condición, dado que su magnitud es al menos igual o superior a $\mathrm{M}_{\mathrm{s}} 7,0$, como detallaremos más adelante en este trabajo.

\section{FALLA DE BOCONÓ}

La Falla de Boconó (FB) es un accidente transcurrente dextral (Rod, 1956b; Stephan, 1982; Schubert, 1982; 1983; Soulas, 1986; Beltrán, 1994; Giraldo, 1985; Audemard et al., 2000; Audemard and Audemard, 2002; Audemard, 2003b; 2009b; entre otros) que se extiende desde la depresión del Táchira al SW hasta el poblado de Morón en el estado Carabobo al NE (FIGURAS 1 y 2), con una longitud total cercana a los $550 \mathrm{~km}$, surcando longitudinalmente los Andes de Mérida y bordeando por el noroeste la depresión de Yaracuy. En su extremidad sur, la falla se conecta con el sistema de piedemonte llanero colombiano a través del sistema de fallas de ChinácotaBramón, luego de sufrir dos inflexiones en ángulo recto opuestas; estructura conocida como el indentor o punzón de Pamplona (Boinet, 1985; FIGURA 1). Por otra parte, la falla cambia su rumbo en $45^{\circ}$ en forma horaria al norte de Morón, para conectarse con grandes fallas dextrales de dirección este-oeste que corren al norte de Venezuela continental (fallas de San Sebastián y El Pilar; FIGURA 1), hasta extenderse a la falla Warm Spring en Trinidad central, por intermedio del sistema de fallas de Los Bajos-El Soldado (Audemard et al., 2005). La FB actualmente aparenta tener un rol preponderante en la fragmentación de bloques tectónicos mayores y en la tectónica de escape imperante en el noroeste de Suramérica, limitando directamente el bloque triangular de Maracaibo por el sureste (FIGURA 1; Audemard and Audemard, 2002; Audemard, 2003b; 2009b).

Dentro de los Andes de Mérida, la FB está siempre realzada por el alineamiento continuo de grandes valles de diversos ríos con anchura variable entre 1 y
$5 \mathrm{~km}$. Esta falla ha sido reconocida, cartografiada y caracterizada desde el trabajo pionero de Rod (1956b), sobre la base del gran número de evidencias geomórficas de fallamiento activo presentes a lo largo de la totalidad de su traza (e.g., Schubert, 1980; 1982; Soulas, 1985; Soulas et al., 1986; 1987; Casas, 1991; 1995; Audemard 2009a). Las geoformas más frecuentes son: cuellos y ensilladuras de falla, trincheras, lagunas de falla, escarpes de falla, lomos lineales y lomos de obturación a lo largo de su(s) traza(s) activa(s). Por su parte, en el valle del Yaracuy, la falla bordea el pie sureste de la sierra de Aroa en sus dos tercios más meridionales entre Yaritagua y San Felipe-, mientras que en su tercio más septentrional corta diagonalmente el propio valle, entre San Felipe y Morón (FIGURA 3). A pesar que la FB en su segmento San Felipe (Boc-e; FIGURA 2), a lo largo de su traza activa, está igualmente expresada por el mismo conjunto de geoformas antes nombradas en el sector andino (FIGURA 3), la falla aquí coincide con un desnivel morfoestructural muy marcado en sus $2 / 3$ suroccidentales, representado por la falda sureste de la sierra de Aroa que alcanza hasta unos $13^{\circ}$ de pendiente y desniveles topográficos de hasta unos $1500 \mathrm{~m}$ en su sector más elevado, en proximidad a la ciudad de San Felipe. En este sector adosado a la sierra de Aroa, la FB sigue un rumbo NE, pero en los alrededores de Albarico, ubicado a unos $6 \mathrm{~km}$ al NE de San Felipe, la falla adquiere una orientación ENE-OSO para cruzar el valle de Yaracuy diagonalmente desde una margen hasta la opuesta (FIGURA 3). En este tramo más septentrional, la FB se expresa por un escarpe de falla de exposición norte (FIGURA 3), de hasta unos $20 \mathrm{~m}$ de alto en proximidad a Albarico, preservado en sedimentitas continentales de color crema, adscritas al Pleistoceno.

Aunque este accidente tectónico puede seguirse casi ininterrumpidamente sobre una longitud próxima a $550 \mathrm{~km}$ sobre la base de los criterios morfológicos antes mencionados, la FB no es un rasgo continuo desde el punto de vista tectónico, tal como lo plantean Audemard et al. (2000), al subdividirla en cinco secciones, preliminarmente distinguidas por subíndices alfabéticos ("Boc-a" a "Boc-e"; FIGURA 2). El criterio para su subdivisión por ellos empleado, reposa esencialmente en irregularidades o discontinuidades de orden geométrico, que concuerdan mayoritariamente con estructuras tectónicas reconocidas como cuencas en tracción (de Lagunillas - explorada sus fallas limítrofes por las trincheras T10 y T11-, Apartaderos - explorada por T3 y T5- y Cabudare -al sur de Barquisimeto-, del SO al NE, en FIGURA 2); a excepción de la barrera entre los segmentos Boc-c y Boc-d, en proximidad a la población de Boconó, que es de tipo transpresivo 
(FIGURA 2). En igual sentido, se puede aseverar que tampoco es un único rasgo en lo sismogenético. Del análisis de los grandes sismos históricos andinos, como en su porción más septentrional en el valle del Yaracuy, se puede percibir que las isosistas VIII o superiores (FIGURA 2), así como los epicentros macrosísmicos propuestos para muchos de ellos no coinciden geográficamente (FIGURA 2; e.g., Soulas et al., 1987 en Audemard, 1997; Grases, 1990; Palme y Altez, 2002; Palme et al., 2005a; Choy et al., 2010); por lo que no pueden ser considerados repeticiones unos de otros. Por ende, la combinación de ambos aspectos -geométricos y sísmológicos- permitía anticipar la posible segmentación sismogenética de la FB, donde cada sección originalmente propuesta por Audemard et al. (2000) pudiese comportarse como un segmento con una historia sísmica propia, por lo que Audemard (2001) planteó corroborar esta hipótesis a través de la excavación de una serie de trincheras de evaluación paleosísmica estratégicamente ubicadas.

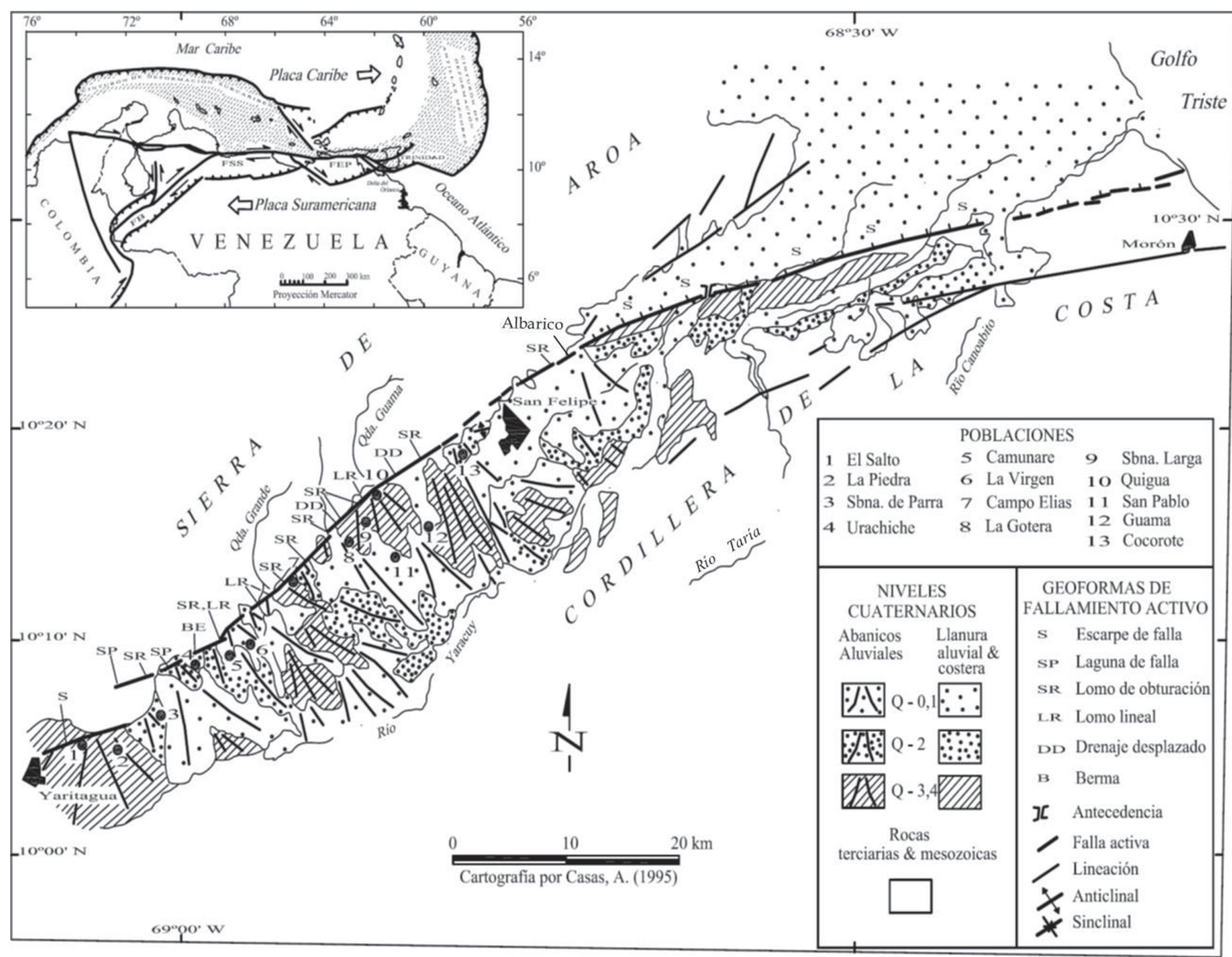

FIGURA 3. Cartografía morfotectónica de la Falla de Boconó, así como de las unidades sedimentarias cuaternarias principales en el valle del Yaracuy enmarcadas por rocas pre-cuaternarias (según Casas, 1995). También se indican las principales localidades nombradas en el texto.

Variaciones en la tasa de movimiento a lo largo de la FB, considerado como otro de los criterios para segmentar fallas (referirse a Audemard and Singer, 1996), también soportan esa segmentación. El desplazamiento lateral derecho de complejos morrénicos del Último Máximo Glacial (localmente nombrado Glaciación Mérida Tardía por Schubert, 1974) en la región de Apartaderos, permiten estimar la tasa de desplazamiento de la FB que limita la cuenca en tracción de Apartaderos durante el Cuaternario tardío en 2,3-3,0 $\mathrm{mm} / \mathrm{a}$ para el ramal norte (terminación noreste del segmento Boc-b), y en 5,0-7,7 $\mathrm{mm} / \mathrm{a}$ para el sur (terminación suroeste del segmento Boc-c), representando aproximadamente el 25 y $75 \%$ de los 7 a $10 \mathrm{~mm} / \mathrm{a}$ de la tasa de desplazamiento total de la FB en los últimos $15 \pm 2 \mathrm{ka}$ (Audemard et al., 1999a). Esta tasa total de movimiento es esencialmente 
consistente con la velocidad de $10 \mathrm{~mm} / \mathrm{a}$ predicha por los modelos de placas tectónicas, asumiendo que la FB es el límite de placas entre el Caribe y Sudamérica (e.g., Molnar and Sykes, 1969; Minster and Jordan, 1978), a partir de marcadores geológicos (e.g., Soulas, 1986), y de mediciones vectoriales más recientes de Posicionamiento Global (e.g., Freymueller et al., 1993; Trenkamp et al., 2002; Pérez et al., 2011). De este valor máximo en la región central andina, la velocidad de la FB decrece hacia sus extremos. Hacia el SO, la tasa de desplazamiento promedio desciende a 5,2 \pm $0,9 \mathrm{~mm} / \mathrm{a}$ entre Mérida y San Cristobal (Audemard, 1997) y a tanto como a $1 \mathrm{~mm} / \mathrm{a}$ en la frontera colombovenezolana (Singer and Beltrán, 1996). De igual manera, ramificaciones de la falla y fallamiento activo subaparalelo podrían ser la causa de la pérdida de velocidad de la FB (1,5-3 mm/a) reportada por Casas (1991), en el valle del Yaracuy.

\section{SECCION BOC-E}

A continuación se describen las principales características morfológicas y evidencias de fallamiento activo a lo largo de la sección San Felipe (Boc-e, según nomenclatura de Audemard et al., 2000) de la $\mathrm{FB}$, discriminadas en tres tramos principales, de NE a SO (FIGURA 3): Golfo Triste-Albarico, Albarico-La Virgen y La Virgen-Yaritagua.

\section{Tramo golfo Triste-Albarico}

En el sector más septentrional de la FB, la traza de la falla adquiere una peculiar orientación ENE-OSO (FIGURA 3). Este tramo permite la conexión de la FB ubicada a lo largo del pie de la sierra de Aroa de orientación NE-SO -rumbo característico presente en todo el resto de su extensión hasta la frontera colombo-venezolana, por unos $500 \mathrm{~km}$ (FIGURAS 1 y 2)-, con la falla de San Sebastián de tendencia este-oeste, cuya traza es esencialmente submarina (FIGURA 1) y se extiende entre golfo Triste en el oeste y la fosa de Cariaco en el este. Entonces, la FB en este sector, cruza el valle de Yaracuy de orientación NE-SO, diagonalmente desde la vertiente sureste de la sierra de Aroa, en proximidad a Albarico (ubicado a unos $6 \mathrm{~km}$ al NE de San Felipe), hasta acercarse a la margen opuesta pero sin alcanzarla (FIGURA 3). Aquí la FB se expresa por un escarpe de falla de exposición norte muy conspicuo, continuo sobre varios kilómetros de longitud, y ocasionalmente por tramos tan extensos como unos $10 \mathrm{~km}$ (FIGURA 3), de hasta unos $20 \mathrm{~m}$ de alto en proximidad a Albarico, preservado en sedimentos continentales pleistocénicos de color crema. El escarpe de falla en su extremo ENE, aproximándose a la costa del golfo Triste, corta rocas sedimentarias más viejas, adscritas al Terciario (FIGURA 3). En este sector, el escarpe limita un bloque, donde el fondo del valle de Yaracuy aparenta estar muy levemente basculado hacia el SSE, hacia el relieve de la Cordillera de la Costa. La red de drenaje principal de la zona que drena la vertiente norte de la Cordillera de La Costa, incluyendo al río Taría y todos al este de él (río Canoabito y Urama) están controlados por este basculamiento, tal como lo denota sus largos segmentos de curso que corren subparalelos a éste y entre la superficie basculada y las faldas de la Cordillera de La Costa (FIGURA 3). Por otra parte, el río Canoabito y sus afluentes están dispuestos en rastrillo, tal como lo describe Audemard (1999) y Ollarves et al. (2006), tendiente a aumentar su caudal para finalmente lograr cruzar este basculamiento y su escarpe limítrofe (FIGURA 3; tasa de erosión del río Canoabito < tasa de movimiento vertical de la falla).

El curso del río Yaracuy también es anómalo. En su tramo superior, este río se recuesta al pie de la Cordillera de la Costa, hasta una fuerte inflexión que lo reorienta norte-sur, al este de la ciudad de San Felipe (FIGURA 3). A pesar que el río Yaracuy se aproxima a la traza activa de la FB, sólo logra cruzar el escarpe unos 3 a 4 $\mathrm{km}$ más al este, luego de correr paralelo a la FB por esa distancia (FIGURA 3). Ello implica que el río Yaracuy es antecedente al escarpe de falla y que su caudal fue lo suficiente importante, aunque no ampliamente superior, para siempre imponerse a la actividad de la FB (tasa de erosión $\geq$ tasa de movimiento vertical de la falla). Ya en el bloque norte de la FB, el río Yaracuy se recuesta contra el pie del escarpe de exposición norte, en posición de gotera tectónica (FIGURA 3), tal como descrito por el autor en Audemard (1999). Esto implicaría que el bloque norte está basculado hacia el sur también, por lo menos en proximidad a la falla, pudiéndose invocar 2 mecanismos distintos: a) simple basculamiento asociado a una componente normal de movimiento vertical de la FB o, b) por el contrario, una pequeña depresión flexural por sobrecarga del bloque sur de la FB sobre el norte, implicando componente secundaria vertical en falla inversa en la FB en este sector), siendo esta última la opción por la cual nos inclinamos.

\section{Tramo Albarico-La Virgen}

En este sector como el contiguo hacia el SO, la traza de la FB se encuentra bien definida al pie del relieve de rocas metamórficas de la sierra de Aroa, que limita el valle de Yaracuy al NO (FIGURA 3). Este tramo presenta las mejores evidencias de actividad tectónica 
reciente de todo el tramo en estudio. La continuidad de las características morfológicas permite identificar la traza de la falla y las deformaciones asociadas de forma clara.

De NE a SO, entre la ciudad de San Felipe y la población de Quigua (10 en la FIGURA 3), existen depósitos y abanicos cuaternarios fallados, drenajes desplazados en unas pocas decenas de metros, diques naturales que represan el curso del drenaje con depósitos cuaternarios correlativos, crestas estiradas en sentido dextral, y una gran variedad de facetas triangulares que van desde 30 $\mathrm{m}$ de altura al NE de la población de Cocorote (13 en la FIGURA 3), hasta varias decenas de metros al norte de la población de Quigua.

Hacia el SO de Cocorote, la FB se expresa en una traza única pero discontinua hasta la población de Quigua, donde la FB se ramifica en varias trazas subparlalelas. Entre ellas una resalta en particular, en el sector conocido como Hacienda El Playón, ubicado justo al este de Quigua, donde la FB se encuentra definida por un escarpe y un contraescarpe de $1,5 \mathrm{~m}$ de alto preservado en un abanico de escombros cuaternario (FIGURA 4), el cual a su vez tiene su eje desplazado dextralmente en unos $60 \mathrm{~m}$ aproximadamente, mientras un drenaje muy menor sólo exhibe un desplazamiento de unos $7 \mathrm{~m}$ (FIGURA 5). Ésta es una de las 2 localidades finalmente evaluadas con fines paleosísmicos, a pesar de exhibir trazas subparalelas aunque con indicios de menor actividad.

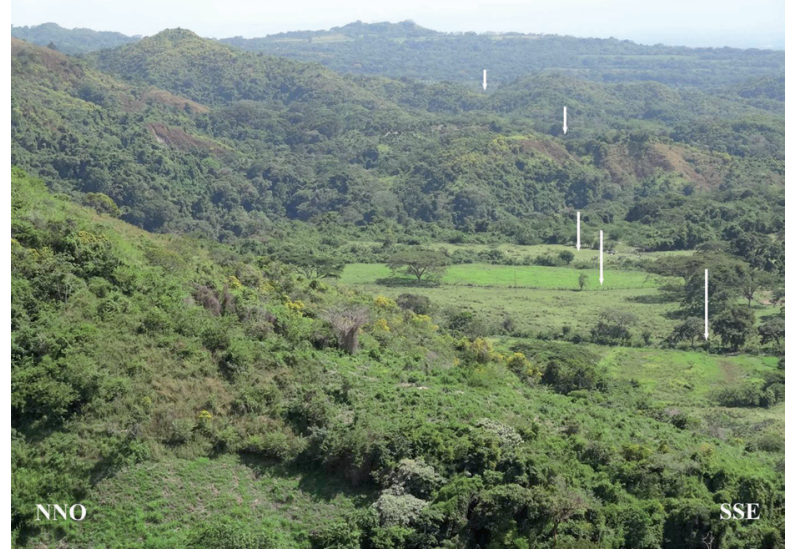

FIGURA 4. Vista oblicua del abanico de Quigua, en la Hacienda El Playón, en dirección del rumbo de la FB, donde se realza la posición de la traza activa por medio de flechas. La ubicación de la tercera flecha (en posición central entre todas) indica aproximadamente donde se excavó la trinchera de Quigua. La segunda flecha más cercana señala un contraescarpe (explicación en el texto). En primer plano, se aprecia el perfil de una faceta triangular bien degradada.

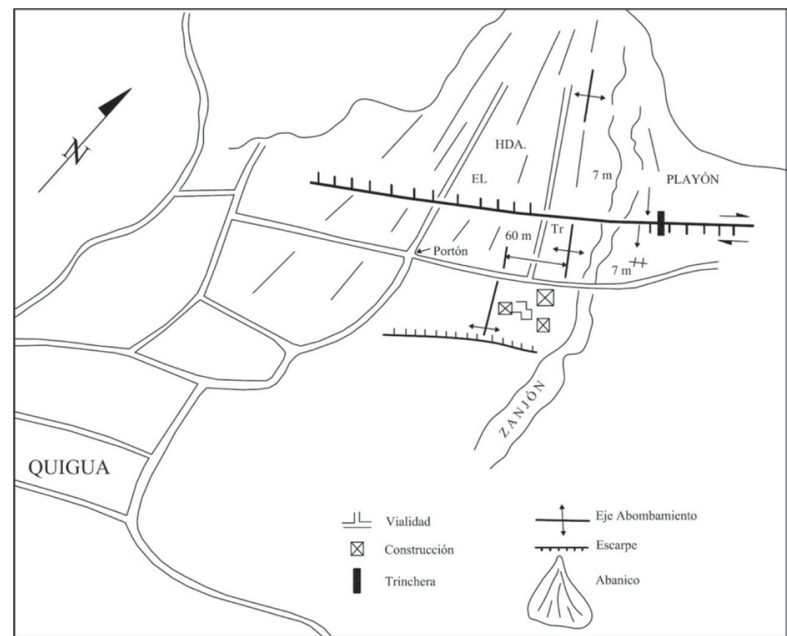

FIGURA 5. Dibujo simplificado del abanico de Quigua, en vista oblicua hacia el NNO, en la Hacienda El Playón, donde se señalan las geoformas que identifican la traza activa de la $\mathrm{FB}$, como las que sustentan su cinemática dextral en esta localidad. Nótese el desplazamiento hacia la derecha del eje del abombamiento del abanico en $60 \mathrm{~m}$, como de pequeños cursos de agua intermitentes que surcan su superficie en sólo $7 \mathrm{~m}$.

Continuando hacia el SO, entre Quigua hasta las proximidades de Campo Elías (10 y 7 respectivamente, en la FIGURA 3), la traza principal de la FB continúa bordeando la sierra de Aroa. Al norte del poblado de Sabana Larga (9 en la FIGURA 3), la falla está definida por una trinchera de $250 \mathrm{~m}$ de ancho aproximadamente entre dos trazas principales y sus respectivos escarpes.

En las cercanías de La Gotera (8 en la FIGURA 3; 4 km al SO de Quigua), la expresión morfológica de la falla es muy clara aunque muestra poca continuidad, y se compone de múltiples trazas subparalelas, pero en una franja inferior a $1 \mathrm{~km}$ de ancho. Cabe resaltar la presencia de lomos de presión, que aparentan haber funcionado como lomos de obturación, detrás de los cuales se represaron depósitos cuaternarios. También se aprecian en el sector trincheras de falla, facetas triangulares al pie del relieve y drenajes desplazados en sentido dextral.

Finalmente, al acercarse a Campo Elías (7 en la FIGURA 3), la FB se muestra en una traza única, muy continua y con buena expresión morfológica, que se extiende hasta las cercanías de La Virgen (6 en la FIGURA 3), entre cuyas evidencias geomórficas se pueden señalar: drenajes desplazados dextralmente, crestas estiradas y alineadas de forma sub-paralela a la traza de la falla, drenajes desplazados alrededor de $800 \mathrm{~m}$ de manera dextral, y un escarpe de falla muy continuo, con exposición sur y magnitud considerable (10 m aprox.) que limita el asentamiento de Campo Elías hacia el NO. 
En las márgenes de las quebradas situadas al SO de Campo Elías, se identificó deformación frágil en secuencias aluviales con buena estratificación y en algunos casos represamiento de material aluvial contra un lomo de obturación, que temporalmente bloqueó el drenaje producto de la cinemática de la falla.

En las adyacencias de la poblacion La Virgen (6 en la FIGURA 3), la traza principal pierde continuidad y expresión morfológica al acercarse al relieve metamorfico de la sierra de Aroa, donde prácticamente desaparece, para reaparecer por medio de un escarpe de falla aproximadamente $1 \mathrm{~km}$ más al $\mathrm{SO}$, bordeando el relieve ubicado al NE de la población de Camunare. En este sector la traza de la falla esta subrayada por facetas triangulares consecutivas de $10 \mathrm{~m}$ de altura en promedio, con exposición al SE y la presencia de un lomo de obturación de magnitud discreta y situado en depósitos aluviales recientes.

Al norte de la población La Virgen, se evidencian una serie de terrazas, asociadas a la Quebrada Cocorotico, que se encuentran basculadas a contrapendiente hacia el NO. Es importante señalar, que aunque el origen de estas terrazas puede ser producto de esfuerzos tectónicos, su registro y preservación puede responder a cambios climáticos, tal como ocurre para las terrazas del flanco surandino (Audemard, 1999; Audemard et al., 2007; Guzmán et al., 2013). Sin embargo, la surrección en el Cuaternario de la sierra de Aroa parece estar más fuertemente sustentada por la geometría y disposición de los depósitos cuaternarios del valle del Yaracuy, así como por la posición excéntrica del río Yaracuy en este tramo, al igual que en el tramo más SO del segmento Boc-e (San Felipe) de la FB (FIGURA 3). Como ha sido ya mencionado, el río Yaracuy corre, entre Yaritagua y más al NE de San Felipe, adosado al pie de la Cordillera de La Costa, a consecuencia del empuje ejercido por los prominentes depósitos de abanicos de múltiple generación que descienden de la sierra de Aroa (FIGURA 3). Casas (1995) cartografía al menos 4 niveles de abanicos (FIGURA 3) e indica que están esencialmente constituidos de cantos y gravas de rocas metamórficas en una matriz arenosa.

Con base en sus estructuras sedimentarias, este autor determina que son transportados en sus porciones medias y distales por cursos de agua, mientras que sus áreas proximales parecen mas bien dominadas por flujos de escombros contentivos de esquistos alterados de las formaciones Aroa y Nirgua. Igualmente, Casas (1995) determina que los distintos niveles de abanicos están escalonados, estando encajados los más jóvenes en los más antiguos, donde el nivel más viejo es el más disectado y elevado, con remanentes aislados (FIGURA 3). Es decir, a medida que el abanico es más joven es altitudinalmente más bajo y mejor preservado (tanto al nivel de la superficie del abanico como de su extensión areal). En las zonas distales, los distintos niveles de abanicos tienden a converger o los más jóvenes solapan a los anteriores, según este mismo autor. En resumidas, las superficies de los abanicos están en tijeras, implicando que la sierra de Aroa ha estado en levantamiento durante el Cuaternario, tal como determina Audemard (1999) para los Andes de Mérida en el flanco surandino, sobre la base del mismo criterio. No obstante, Casas (1995) indica que en algunos casos en estas zonas distales del valle del Yaracuy, el lugar de depositación se encuentra desplazado aguas abajo, requiriendo que la red de drenaje socave los niveles de abanicos anteriores, lo cual Bull $(1977 ; 1984)$ interpreta como una desaceleración de la tasa de levantamiento del relieve de donde se alimentan estos abanicos.

\section{Tramo La Virgen-Yaritagua}

De manera general, este tramo de la FB se caracteriza por poca linearidad y una marcada complejidad estructural de su traza principal, aunque la expresión morfológica de la(s) traza(s) se limita muy frecuentemente a escarpes de falla disectados en facetas triangulares al pie del relieve, y a algunos abanicos y drenajes desplazados. En proximidad a La Virgen-Camunare (6 y 5 respectivamente, en FIGURA 3 ), la traza principal presenta un salto senestro, pero el más notorio es el ubicado entre Sabana de Parra y Urachiche (3 y 4 respectivamente, en FIGURA 3). Aquí, la traza se ramifica nuevamente, delimitando un relieve prominente, con forma en planta de almendra, que asemeja una gran estructura pop-up (FIGURA 6), donde las fallas más internas son más lineales en su trazado mientras el de la falla que corre a lo largo de su flanco oriental (y limítrofe de la depresión del Yaracuy) se muestra más alabeado, sugiriendo que su buzamiento tiende a valores intermedios. La morfología tiende a soportar la presencia de una estructura en flor positiva, en este gran solape senestro que se extiende entre las poblaciones El Salto y Urachiche (1 y 4 respectivamente, en FIGURA 3).

Al norte de Urachiche, la traza principal de la FB se ubica al pie del relieve y concentra las evidencias de actividad reciente por medio de un escarpe de falla de $8 \mathrm{~m}$ de altura aproximadamente y con muy buena continuidad lateral, que se expresa justo en los linderos norcoccidentales de esta población. Particularmente, en el extremo NE del poblado de Urachiche, existe un afloramiento en la margen derecha de la quebrada 
Cocorotico, que expone deformación frágil asociada a la traza principal de la FB, la cual es apreciable desde el cauce de la quebrada, el plano de falla es sub-vertical con acimuth medido de $\mathrm{N} 100^{\circ}$, y en superficie se manifiesta por un pequeño escarpe de falla. Conjuntamente, en el extremo oeste del pueblo, en el sector de Sabana de Guachire, la traza activa de la falla se evidencia por una laguna de falla de $7 \mathrm{~m}$ de diámetro, represada contra un lomo de obturación de escasos metros de desnivel.

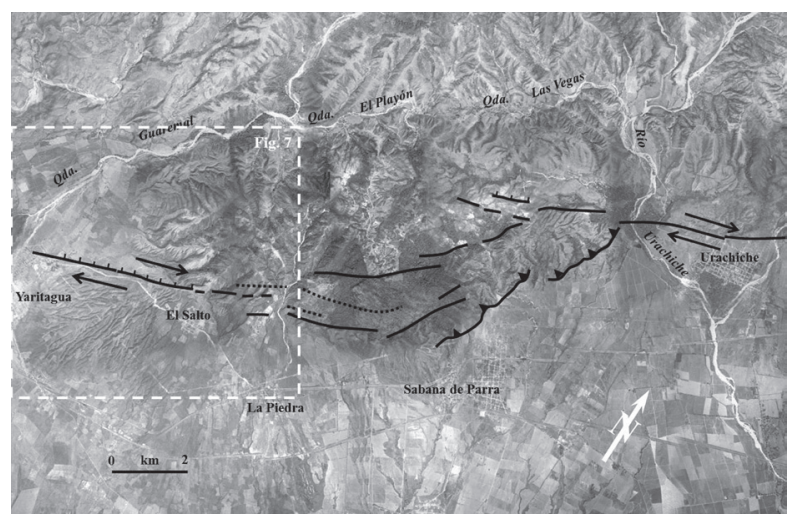

FIGURA 6. Mosaico de vistas aéreas de la misión 020317 (Cortesía del Instituto Cartográfico Venezolano Simón Bolívar -IGVSB-), donde se muestra un relevo transpresivo de grandes dimensiones entre Yaritagua y Urachiche. Se señala igualmente la ubicación relativa de la FIGURA 7.

Más al SO, en las adyacencias del río Urachiche, la traza principal afecta depósitos cuaternarios muy recientes originando un escarpe de magnitud discreta y exposición norte. Luego, la falla continúa en la margen derecha del drenaje expresada por una pequeña trinchera de falla y escarpes de dimensión reducida. Prosiguiendo hacia el SO, la falla continúa como una traza contínua que se encaja en el relieve con aspecto de "pop-up" y es evidenciada como una trinchera de falla, con algunos escarpes y contraescarpes de exposición norte y sur respectivamente. También se observan en el sector fallas secundarias sub-paralelas a la traza principal, expresadas por facetas triangulares y pequeños escarpes (FIGURA 6).

Al norte de Yaritagua, la componente de rumbo de la Falla de Boconó genera un contra-escarpe rectilíneo (descrito inicialmente por Schubert y Krause, 1981), que desplaza el abanico de Yaritagua en sentido dextral y lo desconecta de la fuente potencial de aporte de sedimentos (FIGURA 7). Casas (1991) estimó un desfase horizontal de al menos $1,2 \mathrm{~km}$. El escarpe en este sector tiene una altura promedio de $10 \mathrm{~m}$, cuya magnitud decrece hacia el SO. En el extremo superior del abanico, se identificó una trinchera de falla de $12 \mathrm{~m}$ de ancho y $1000 \mathrm{~m}$ de largo generada por la traza única de la falla en la zona. Cabe destacar que en la parte NE del escarpe se evidencian una serie de estructuras que represan el curso de algunos drenajes intermitentes en la zona que se encuentran desplazados en sentido dextral y se alinean en dirección del rumbo de la falla. Al norte, cerca de la naciente de la quebrada El Salto (al NE de la población de mismo nombre) existen una serie de drenajes descabezados que han sido desconectados de su fuente de aporte producto de la cinemática dextral de la falla.

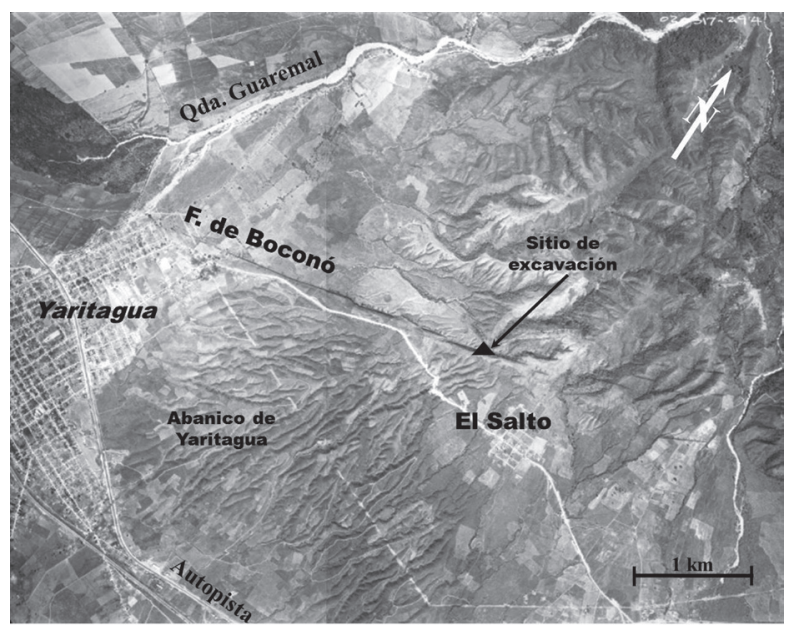

FIGURA 7. Vista aérea de la misión 020317, mostrando el abanico de Yaritagua desconectado parcialmente del relieve y desplazado dextral por la FB (Cortesía del Instituto Cartográfico Venezolano Simón Bolívar-IGVSB-). Se señala la ubicación de la excavación paleosísmica denominada Yaritagua, próxima a la localidad de El Salto.

\section{EL ÚLTIMO SISMO EN EL SEGMENTO BOC-E: EL SISMO DEL 26-03-1812}

Como indicado previamente, las exploraciones paleosísimicas ejecutadas en este segmento Boc-e de la $\mathrm{FB}, \mathrm{y}$ que aquí presentaremos más adelante, fueron dirigidas particularmente a determinar la asociación sismotectónica del sismo del 26 de marzo de 1812. En consecuencia, particular atención le vamos a dedicar a este terremoto en lo sucesivo.

Diversos autores (Centeno Graü, 1969; Fiedler, 1961; 1972; Rodríguez y Audemard, 1997; Audemard, 2002; Altez, 2005a y b) han considerado al sismo del 26 de marzo de 1812 como un sismo multifoco. Centeno Graü (1969) considera que tuvo tres focos: uno en el mar Caribe, entre los Roques y la costa de La Guaira; otro frente a San Felipe, Barquisimeto y El Tocuyo; y un tercero, al sur del lago de Maracaibo. Fiedler (1961; 1972) también apoya la hipótesis de que tuvo tres focos: 
uno al norte de la costa de la Guaira, un segundo foco en la Falla de Boconó, entre San Felipe y Barquisimeto, y un tercero al suroeste de Mérida. Altez (2005a; 2005b; 2006) sostiene que en realidad tuvo dos focos, ya que considera que el movimiento sísmico que afectó a Mérida ocurrió aproximadamente una hora después de los de Caracas y San Felipe. En tal sentido, Rodríguez y Audemard (1997), posteriormente publicado por Altez (1998), plantea la idea de la existencia de dos sismos diferentes, en la misma tarde y a diferentes horas, para las regiones de Caracas-Barquisimeto y Mérida, respectivamente. En el trabajo de FUNVISIS (Rodríguez y Audemard, 1997) se presentan dos mapas de isosistas que separan los efectos de los dos sismos. Esta nueva hipótesis se sostiene en la evidencia documental que demuestra que Caracas fue afectada por un terremoto a las 16:07, mientras que en Mérida la afectación ocurrió alrededor de las 17:00 horas (ambas horas locales; no hubo homologación horaria en Venezuela hasta 1912) del mismo 26 de marzo. Esta es la hipótesis actualmente más compartida, aceptándose que en realidad son dos eventos independientes, sólo separados por menos de una hora, los que afectan, por una parte, a la región central y centro-occidental del país y, por otra parte, casi exclusivamente a la ciudad de Mérida; siendo este segundo un evento de magnitud moderada por lo pequeño de su área sentida, pero con muchos efectos de sitio y de amplificación por el espesor de la terraza de Mérida. Por el contrario, el evento que afectó Barquisimeto, San Felipe y Caracas principalmente es un evento mayor que probablemente resulta de una ruptura compuesta, con por lo menos dos focos (uno en el mar en el centro-norte del país y otro en tierra en el valle del Yaracuy; FIGURA 2).

Fiedler (1961) le asigna una magnitud de ondas superficiales $M_{s}=6,3$ al sismo de Caracas; $M_{s}=6,2$ al de San Felipe y de 7,0 al de Mérida. Posteriormente, Fiedler (1972) realiza una reevaluación y asigna una magnitud de ondas de cuerpo $\mathrm{mC}=7,1$ a cada uno de los tres eventos, que corresponde a una magnitud $\mathrm{M}_{\mathrm{s}}=$ 7,3 (para esta equivalencia véase Richter, 1958). Grases (1990) estima en 7,0, 7,2 y 6,3 las magnitudes para las rupturas en Mérida, Barquisimeto-San Felipe y Caracas, respectivamente. Grases y Rodríguez (2001), a partir de una relación por ellos derivada entre magnitud $\mathrm{M}_{\mathrm{s}}$ y el área inscrita por cada isosista, proponen valores de 6,56,7; 6,9-7,2 y 7,1 para los eventos en Mérida, San Felipe y Caracas, respectivamente. Audemard (2002) quien estudia las rupturas de los grandes sismos históricos a partir del análisis de la sismicidad contemporánea (aplicando el concepto de silencio sísmico), propone para el subevento de Caracas una ruptura de la falla de San Sebastián con un foco que se encuentra en una posición más intermedia entre Puerto Cabello y La Guaira y al oeste del propuesto por Fiedler, sin hacer inferencias sobre su magnitud. Igualmente, Audemard (2002) indica que debería ubicarse al oeste de la ruptura del sismo de Caracas del 29 de julio de 1967 de magnitud 6,3 que ocurriría más tarde para completar una secuencia sísmica conjuntamente con los sismos de 1812 y 1900, y geográficamente intercalada entre las rupturas de ambos eventos. Para el segundo foco, este mismo autor propone como fuente la terminación norte de la FB a lo largo del valle de Yaracuy que se extiende desde la cuenca de tracción de Cabudare hacia el NE. La magnitud de este segundo subevento la estima en aproximadamente 7,0 a partir de la extensión de la isosista VIII. En referencia al segundo sismo, Laffaille y Ferrer (2003) consideran que la ruptura de Mérida tuvo una magnitud $\mathrm{M}_{1} 5,1-5,4$ y que la falla del Albarregas (Laffaille y Ferrer, 2005) podría haber estado asociada con el evento sísmico.

Para las evaluaciones del terremoto de 1812 todos los autores, salvo Audemard (2002), han utilizado isosistas, locualimplicainferencias "visuales", que potencialmente pueden introducir sesgos y hacen difícil realizar verificaciones estadísticas (Gasperini et al., 1999). Para tratar de obtener inferencias más objetivas a partir de intensidades de sismos históricos, Bakun and Wenworth (1997; 1999) desarrollaron un método estadístico que utiliza directamente las observaciones individuales de intensidades en la determinación de epicentros y magnitudes. El método requiere de un proceso previo de calibración entre intensidades, magnitudes y distancias epicentrales, lo cual se logra a través de sismos modernos registrados instrumentalmente (Choy et al., 2010). Estos autores también señalan que este método igualmente presenta ciertas ventajas con respecto al tradicionalmente utilizado método de las isosistas: (1) Es objetivo en el análisis de las intensidades, sobre todo cuando la distribución de las mismas no es uniforme o su número es escaso. (2) Todos los datos de intensidades contribuyen con el mismo peso al cálculo de la magnitud y del epicentro, y no solamente los cercanos al foco. (3) El tratamiento estadístico de los datos permite establecer intervalos de confianza. Palme et al. (2005b) llevaron a cabo el proceso de calibración para el Centro Occidente de Venezuela. Las relaciones obtenidas fueron utilizadas por Palme et al. (2005a) para analizar la información de intensidades asociada al evento de Mérida, y obtuvieron un centro de intensidades entre las ciudades de Mérida y Tabay, y una magnitud de momento $\mathrm{M}_{\mathrm{wI}} 6,5 \pm 0,5$. Este valor de magnitud solapa con el intervalo obtenido por Laffaille y Ferrer (2003) ya que convirtiendo sus valores 
a magnitud de momento, se obtiene $\mathrm{M}_{\mathrm{wl}} 5,6-6,0$, aunque el intervalo de valores probables obtenido por Palme et al. (2005a) también acepta magnitudes algo mayores.

En años recientes, ha mejorado notablemente la disponibilidad de información sobre los daños causados por los subeventos de Caracas y de San Felipe en 1812, gracias a las investigaciones de RogelioAltez(Altez, 2006) y parcialmente en los catálogos de Grases et al. (1999) y Palme et al. (http://www.sismicidad.ciens.ula.ve), lo cual permitió a Choy et al. (2010) incrementar el número de localidades a las cuales se les pudo asignar intensidades, y además, establecer rangos de incertidumbres para las intensidades previamente reportadas.

Choy et al. (2007) para aplicar el método de Bakun and Wentworth (1997) al sismo de Caracas del 26 de marzo de 1812 (el cual excluye el sismo de Mérida del mismo día), tuvieron que introducirle dos modificaciones por ellos desarrolladas: a) establecer intervalos de intensidades para contemplar todas las incertidumbres derivadas de la propia información histórica; y b) una corrección que considera el rumbo de la falla generadora, mas no su posición espacial. Los principales resultados para cada subevento, arrojados por el estudio de Choy et al. (2010), pueden resumirse a continuación: 1) el subevento Caracas: el centro de intensidades hallado fue $10,60^{\circ} \mathrm{N}$ y $67,10^{\circ} \mathrm{O}$ (próximo al norte de Carayaca) y la magnitud obtenida fue $\mathrm{M}_{\mathrm{wI}} 7,10 \pm 0,33$. La longitud de ruptura estimada es de unos $70 \mathrm{~km}$. La magnitud $\mathrm{M}_{\mathrm{wI}}$ de 7,10 $\pm 0,33$ es consistente con los valores determinados y/o utilizados por otros autores anteriormente mencionados. La ubicación epicentral apunta a una ruptura en la falla de San Sebastián. Por otra parte, es probable que al igual que en 1967, la ruptura haya sido discontinua y que la ruptura haya incluido un segmento de la falla al oeste del epicentro de 1967, tal como lo plantea Audemard (2002). Este autor también plantea que es poco probable que segmentos de la falla de San Sebastián al este del segundo subevento, hayan participado, debido a que esos segmentos rompieron para generar el terremoto del 29 de octubre de 1900; y 2) el subevento BarquisimetoSan Felipe: la magnitud obtenida fue de $\mathrm{M}_{\mathrm{wI}} 7,40 \pm 0,35$, con un centro de intensidades en $10,20^{\circ} \mathrm{N}, 68,95^{\circ} \mathrm{O}$ ubicado entre Barquisimeto y San Felipe. La longitud de ruptura preferida está entre 90 y $100 \mathrm{~km}$ y se le puede atribuir a la FB en su segmento más septentrional.

\section{ESTUDIOS PALEOSÍSMICOS}

En la sección más septentrional de la $\mathrm{FB}$, denominada segmento San Felipe (o Boc-e), que se extiende en dirección NE entre Barquisimeto y Morón, dos evaluaciones paleosísmicas por intermedio de la excavación de las trincheras T8 y T9, fueron realizadas respectivamente en las localidades de El Salto (suburbio noreste de Yaritagua) y Quigua (FIGURA 2).

\section{Trinchera Yaritagua (T8)}

Esta excavación se ubicó en el sector de El Paradero (N10 06'11,8" W69 $05^{\prime} 48,3^{\prime \prime}$ '), entre las poblaciones de Yaritagua y El Salto (FIGURA 7). El sitio seleccionado corresponde a una propiedad privada utilizada con fines de cultivo artesanal, el cual es afectado por un tramo de la FB que se prolonga en forma continua entre las poblaciones de Sabana de Parra y Yaritagua con orientación $\mathrm{N} 60^{\circ} \mathrm{E}$.

En este sitio, la FB presenta una expresión morfológica muy conspícua, correspondiente consecutivamente de $\mathrm{NE}$ a SO a una trinchera de falla y un escarpe de falla de exposición norte (FIGURA 7); este último rasgo morfológico previamente señalado por Schubert (1982). Tales características garantizaron la existencia de simplicidad de la traza activa (FIGURA 7), además de la preservación de materia orgánica y materiales aluviales finamente estratificados (FIGURA 8c); condiciones idóneas para la selección de sitios de excavación paleosísimica (para más detalles, referirse a Audemard, 2003a; 2005). En el sitio particular seleccionado para la ejecución de la excavación, la trinchera de falla corresponde a un dren desactivado por descabezamiento (no corre actualmente agua en su fondo; FIGURAS 8 a $\mathrm{y} b$ ), con forma de bayoneta que registra desplazamiento de su curso en forma dextral. La trinchera fue excavada ortogonal al rumbo de la falla, en forma manual y con dimensiones finales de 8,20 $\mathrm{m}$ de largo, 1,40 $\mathrm{m}$ de ancho y $2,50 \mathrm{~m}$ de profundidad promedio.

Las paredes de la excavación revelan el desarrollo del canal de un río de granulometría francamente granodecreciente, el cual a su vez es cortado por un canal más joven hacia el extremo NNO de la excavación, construido a expensas de canales aluviales más pequeños apilados verticalmente e interdigitados con coluviones fuertemente orgánicos del talud norte del mismo canal (entre 2,5 y $5 \mathrm{~m}$ del levantamiento mostrado en la FIGURA 9). Estos canales más pequeños presentan buena estratificación interna, con variaciones verticales de tamaño de grano, generalmente granodecreciente al interno. Hacia el extremo NNO de la trinchera (4,5-8 $\mathrm{m})$, subyaciendo a un coluvión fuertemente orgánico y conformando el talud, se observó una secuencia de conglomerados estratificados, bien cementados, los cuales parecen pertenecer por sus características físicas al abanico de Yaritagua de edad pleistocena(?). Estos conglomerados se encuentran fallados y separados de 
la secuencia de canales por un nivel orgánico de alta inclinación, el cual es concordante con el escarpe de exposición sur que limita al norte la trinchera de falla (FIGURA 8a).
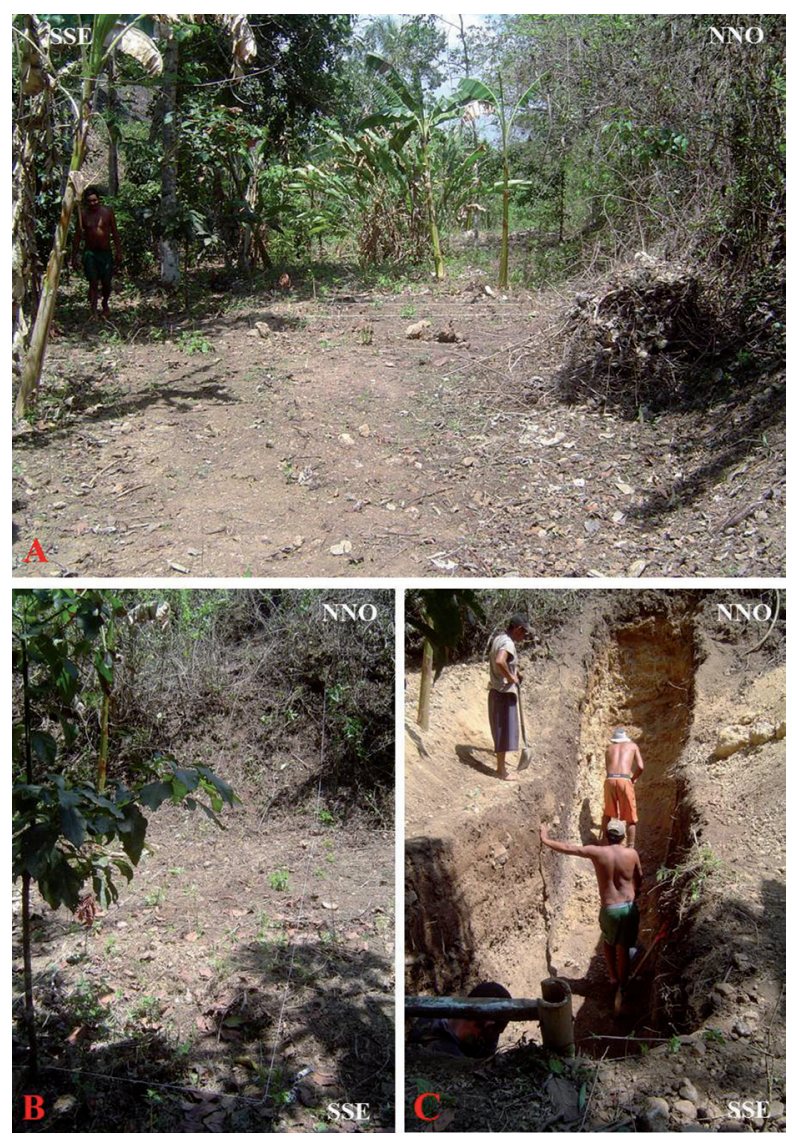

FIGURA 8. Sitio de excavación de la trinchera paleosísmica de Yaritagua. A) Vista hacia el oeste de la trinchera de falla, donde se observa el pie del escarpe de falla de exposición sur. B) Vista hacia el NNO, transversal a la trinchera de falla, donde está demarcada la ubicación del sitio de excavación, en el suelo por medio de hilos blancos. C) Proceso de excavación manual, donde ya se puede apreciar los canales aluviales ricos en materia orgánica en primer plano dentro de la excavación, mientras en segundo plano se aprecian los conglomerados fuertemente cementados de color crema del abanico de Yaritagua.

Del análisis preliminar realizado en trinchera, durante su levantamiento, se estableció que las líneas de cantos (stone lines) preservadas en el coluvión orgánico del talud norte, era correlacionable con la base más gruesa de cada uno de los canales más modestos reconocidos (FIGURA 9). Inicialmente, se interpretó que dichas líneas de cantos (ver Audemard, 2005) representaban antiguos sismos ocurridos en esa localidad, así como un posible control estructural ejercido por la FB sobre la sedimentación de estos paleo-canales; hasta un total de nueve eventos (Ollarves et al., 2005). La otra evidencia interpretada como formada durante un sismo, corresponde a una grieta abierta (open crack, según Audemard, 2005) subvertical y rellena de material orgánico y cantos, que corta en diagonal el coluvión fuertemente orgánico que tapiza el talud norte de la trinchera de falla; la cual fue sólo visible en la pared este (FIGURA 10). Esta grieta abierta, luego de cortar el coluvión orgánico, se enraíza en el contacto basal discordante de este coluvión con los conglomerados pleistocénicos del abanico de Yaritagua de color crema (FIGURA 10).

Afin de datar dicha secuencia de eventos, se recolectaron 18 muestras de niveles orgánicos, y fragmentos de carbón natural, de ambas paredes (FIGURAS 9 y 10), para aplicarles el método del radiocarbono (TABLA 1). Las muestras fueron tomadas en las cuñas coluviales (o líneas de cantos), tal como hiciera Audemard (2006) en las excavaciones realizadas en El Pilar, asumiendo que la matriz orgánica de las mismas fuesen representativas de su edad de depositación. Catorce de estas 18 dataciones radiocarbónicas obtenidas arrojaron ser modernas (> 1950 DC; TABLA 1). No estamos en capacidad de establecer que estas dataciones hayan sido naturalmente contaminadas; o eventualmente rejuvenecidas por sucesivas fases de "tala y quema" practicadas por los agricultores locales -práctica relativamente común en la Venezuela rural, como en otros países latinoamericanos- con fines agrícolas. No obstante, las dataciones $41 \mathrm{y}$ 52 (FIGURA 9; o VEN-41-05 y VEN-52-05 en la TABLA 1), aplicadas sobre fragmentos de carbón y suelo orgánico respectivamente (tener presente que sobre dos materiales distintos), ambas con resultados convergentes, establecieron que el tercio inferior del canal más antiguo es sólo más joven que 1520 DC (FIGURA 9 y TABLA 1); lo cual permite inferir que no hay problemas mayores con las muestras datadas y que verdaderamente la sedimentación observada en la integralidad de la pared resulta ser joven. Por su parte, la pared opuesta (pared este) exhibe también 2 dataciones que resultan distintas a una edad moderna (>1950 DC; FIGURA 10 y TABLA 1). La muestra VEN-43-05, colectada en una de las líneas de cantos más profundas dentro del coluvión (la segunda más profunda después de la muestra VEN-42-05, que resultó igualmente moderna), es de edad similar o apenas más antigua que las muestras 41 y 52 de la otra pared (la oeste; TABLA 1). Esto confirmaría que el canal principal (al menos la sección vertical estudiada de unos $2 \mathrm{~m}$ de espesor) presenta una antigüedad de a lo sumo unos 500 años; y que el canal inciso en el anterior y sus sucesivas fases de relleno granodecrecientes han ocurrido muy recientemente. Lo antes expuesto nos lleva a concluir que los nueve episodios antes identificados 
por Ollarves et al. (2005) deben interpretarse como eventos climáticos de alta lluviosidad, y no son productos de sismo alguno. Esto entra en cierta contradicción con lo reportado con algunos habitantes del sector, quienes señalaron al autor que agua alguna corre por el fondo de la trinchera de falla aún en días de fuerte precipitación. Si esta observación de los lugareños fuese correcta, ¿Qué transporta y deposita los sedimentos fluviales modernos ahí conservados y apreciados en la excavación?

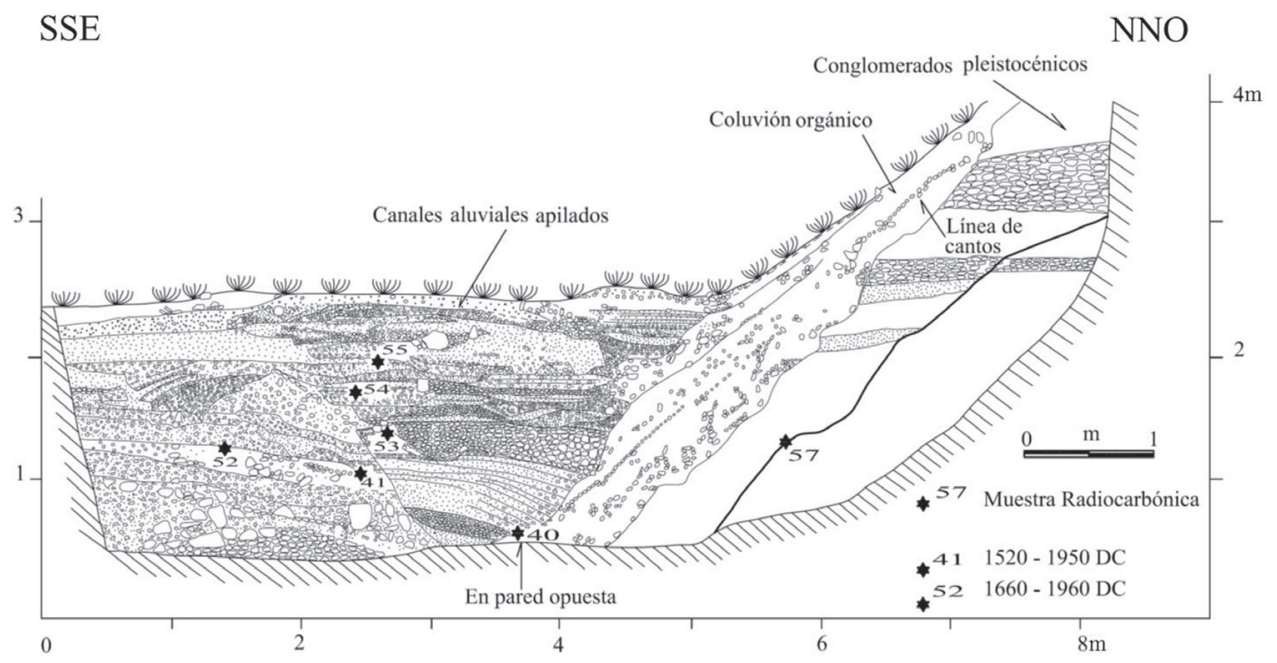

FIGURA 9. Levantamiento geológico de la pared oeste de la trinchera Yaritagua (-T8- en la FIGURA 2). Se identifican todos los puntos de muestreo para datación radiocarbónica, aunque sólo se reportan sólo la edad de dos de ellas. En texto, ver explicación.

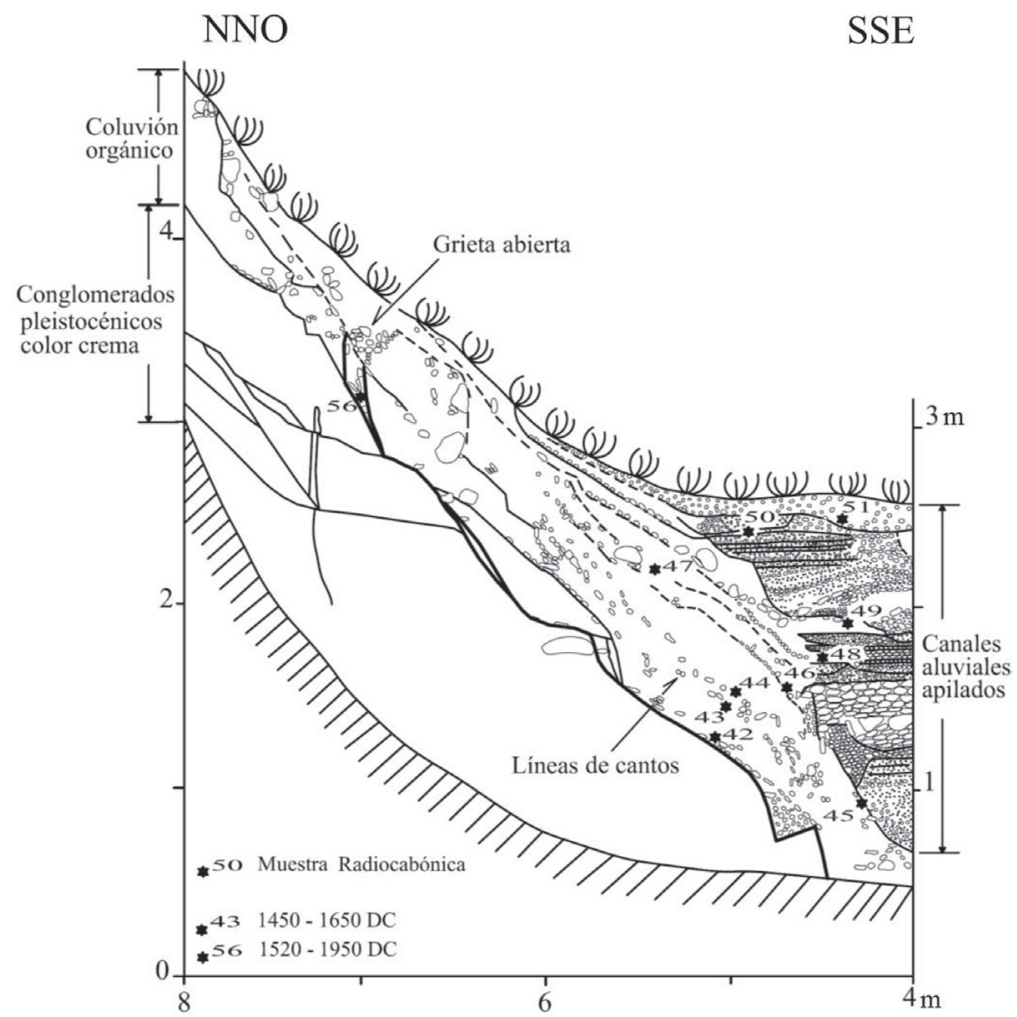

FIGURA 10. Levantamiento geológico parcial de la pared este de la trinchera Yaritagua (-T8- en la FIGURA 2). Se identifican todos los puntos de muestreo para datación radiocarbónica, aunque sólo se reportan sólo la edad de dos de ellas. La evidencia geológica que soporta la ocurrencia del sismo de 1812 en este segmento Boc-e de la FB es la grieta abierta rellena que se halla enraizada en el contacto discordante entre los coluviones ricamente orgánicos y el conglomerado pleistocénico de color crema del abanico de Yaritagua subyacente. 
TABLA 1. Dataciones radiocarbónicas de muestras colectadas en la trinchera Yaritagua (T8). La ubicación de las muestras orgánicas está identificada en los levantamientos geológicos de ambas paredes de la trinchera, mostrados en las FIGURAS 9 y 10.

\begin{tabular}{|c|c|c|c|c|}
\hline \multirow{2}{*}{$\begin{array}{c}\mathrm{N}^{\circ} \\
\text { MUESTRA } \\
\text { FUNVISIS }\end{array}$} & \multirow{2}{*}{$\begin{array}{c}\mathbf{N}^{\circ} \\
\text { BETA } \\
\text { ANALYTIC }\end{array}$} & \multirow{2}{*}{$\begin{array}{c}\text { AÑOS AP CALIBRADOS } \\
2 \text { sigma } \\
\text { (años) }\end{array}$} & \multicolumn{2}{|c|}{$\begin{array}{c}\text { AÑOS CALENDARIO } \\
\text { CALIBRADOS }\end{array}$} \\
\hline & & & 1 sigma & 2 sigma \\
\hline Ven-40-05 & 221855 & $0-0$ & $>1.960$ DC (moderna) & $>1.960$ DC (moderna) \\
\hline VEN-41-05 & 221856 & $\begin{array}{c}430-370 \\
320-270 \\
180-150 \\
10-0 \\
\end{array}$ & $1.640-1.660 \mathrm{DC}$ & $\begin{array}{l}1.520-1.580 \mathrm{DC} \\
1.630-1.680 \mathrm{DC} \\
1.770-1.800 \mathrm{DC} \\
1.940-1.950 \mathrm{DC}\end{array}$ \\
\hline VEN-42-05 & 221857 & $0-0$ & $>1.960$ DC (moderna) & $>1.960$ DC (moderna) \\
\hline VEN-43-05 & 221858 & $500-300$ & $\begin{array}{l}1.460-1.540 \mathrm{DC} \\
1.540-1.630 \mathrm{DC} \\
\end{array}$ & $1.450-1.650 \mathrm{DC}$ \\
\hline VEN-44-05 & 221859 & $\begin{array}{l}630-600 \\
560-510 \\
\end{array}$ & $1.400-1.430 \mathrm{DC}$ & $\begin{array}{l}1.320-1.350 \mathrm{DC} \\
1.390-1.440 \mathrm{DC} \\
\end{array}$ \\
\hline VEN-45-05 & 221860 & $0-0$ & $>1.960$ DC (moderna) & $>1.960$ DC (moderna) \\
\hline VEN-46-05 & 221861 & $0-0$ & $>1.960$ DC (moderna) & $>1.960$ DC (moderna) \\
\hline VEN-47-05 & 221862 & $\begin{array}{c}240-240 \\
60-40 \\
0-0\end{array}$ & $>1.960$ DC (moderna) & $\begin{array}{c}1.710-1.710 \mathrm{DC} \\
1.880-1.910 \mathrm{DC} \\
>1.960 \mathrm{DC} \text { (moderna) }\end{array}$ \\
\hline VEN-48-05 & 221863 & $0-0$ & $>1.960$ DC (moderna) & $>1.960$ DC (moderna) \\
\hline VEN-49-05 & 221864 & $0-0$ & $>1.960$ DC (moderna) & $>1.960$ DC (moderna) \\
\hline VEN-50-05 & 221865 & $0-0$ & $>1.960$ DC (moderna) & $>1.960$ DC (moderna) \\
\hline VEN-51-05 & 221866 & $0-0$ & $>1.960$ DC (moderna) & $>1.960 \mathrm{DC}$ (moderna) \\
\hline VEN-52-05 & 221867 & $290-0$ & $\begin{array}{l}1.670-1.700 \mathrm{DC} \\
1.720-1.780 \mathrm{DC} \\
1.800-1.820 \mathrm{DC} \\
1.840-1.880 \mathrm{DC} \\
1.920-1.950 \mathrm{DC} \\
1.950-1.950 \mathrm{DC}\end{array}$ & $1.660-1.960 \mathrm{DC}$ \\
\hline VEN-53-05 & 221868 & $0-0$ & $>1.960$ DC (moderna) & $>1.960$ DC (moderna) \\
\hline VEN-54-05 & 221869 & $0-0$ & $>1.960$ DC (moderna) & $>1.960$ DC (moderna) \\
\hline VEN-55-05 & 221870 & $0-0$ & $>1.960$ DC (moderna) & $>1.960$ DC (moderna) \\
\hline VEN-56-05 & 221871 & $\begin{array}{c}430-370 \\
320-270 \\
180-150 \\
10-0\end{array}$ & AD 1.640-1.660 & $\begin{array}{l}\text { AD } 1.520-1.580 \\
\text { AD } 1.630-1.680 \\
\text { AD } 1.770-1.800 \\
\text { AD } 1.940-1.950\end{array}$ \\
\hline VEN-57-05 & 221872 & $0-0$ & $>1.960$ DC (moderna) & $>1.960$ DC (moderna) \\
\hline
\end{tabular}

La grieta abierta, correspondiente a la otra evidencia identificada en el coluvión orgánico del talud NNO de la trinchera de falla, entre 8 y $4 \mathrm{~m}$ en FIGURA 10, que reposa discordante sobre los conglomerados del Pleistoceno que conforman el abanico de Yaritagua y contiene todos las líneas de cantos, resultó tener una edad que se extiende, en cuatro periodos discretos (ver TABLA 1), entre 1520 y 1950 DC. Sin embargo, al cortar la línea de canto que arroja una edad entre 1450 y 1650 DC (VEN-43-05 en TABLA 1 y FIGURA 10), el sismo que se evidencia por esta grieta abierta debe ser más joven que 1770 DC. En consecuencia, el evento sísmico que pudiese ser responsable de esta evidencia de ruptura superficial encontrada en la trinchera de Yaritagua debe cumplir con las siguientes condiciones: a) estar asociado a la FB, dado que la trinchera está sobre esta falla; b) presentar una magnitud próxima a $M_{s} 7,0$ para lograr romper la superficie del terreno (por intermedio de grietas abiertas, en este caso), durante un sismo transcurrente; y c) haber ocurrido después de 1770 DC. 


\section{Trinchera de Quigua (T9)}

La excavación más septentrional realizada durante este estudio se ubicó en proximidad a Quigua (FIGURA 2), pequeña población ubicada al ONO de Guama, estado Yaracuy. Ésta se localizó específicamente en la Hacienda El Playón (N $10^{\circ} 17^{\prime} 55,1^{\prime}$; W $68^{\circ} 51^{\prime}$ 18,2"; FIGURA 11). La FB en este sector se extiende en dirección SO-NE, como una traza continua con orientación preferencial $\mathrm{N} 30^{\circ} \mathrm{E}$, desde el norte del poblado de Sabana Larga hasta el NO de la población de Cocorote (FIGURA 3). Presenta una muy clara expresión morfológica, compuesta principalmente por "Riedels sintéticos", los cuales afectan al abanico de Quigua y los drenajes de la zona, que se adaptan a los quiebres generados por la falla.

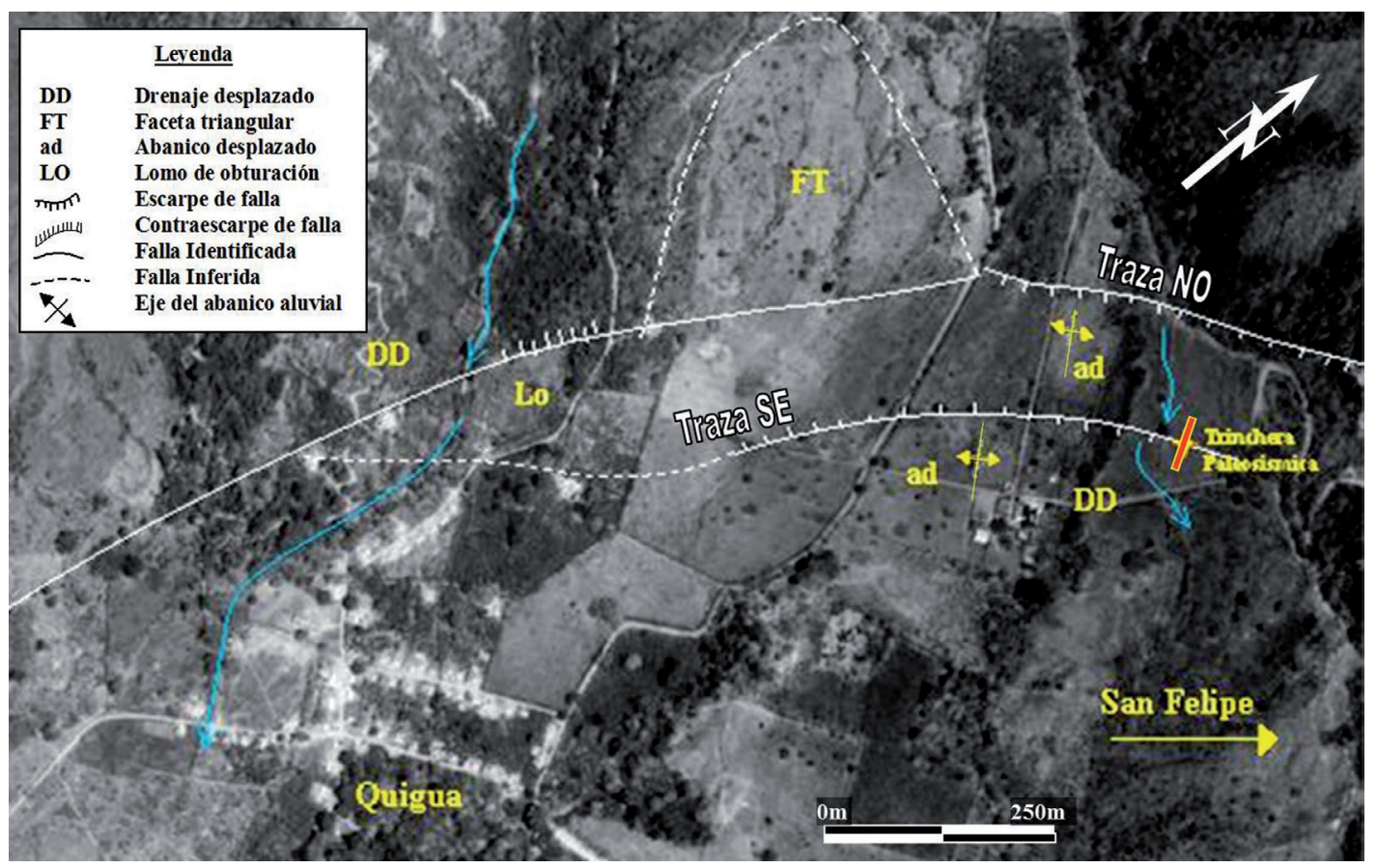

FIGURA 11. Evidencias morfológicas de la FB, en Hacienda El Playón, en proximidad a la población de Quigua (vista aérea 147 de la misión 020317, a escala 1:25.000 del IGVSB), afectando el abanico fluvio-torrencial homónimo. Se señala la ubicación exacta del sitio de excavación paleosísimica denominado aquí como trinchera de Quigua. Comparar con el bosquejo simplificado mostrado en la FIGURA 5.

La FB donde se realizó esta excavación, presenta dos trazas. Una corre al pie del relieve, la cual se expresa por un escarpe de exposición SO que afecta la parte apical del abanico de Quigua (FIGURA 11), denominada "Traza NO". La segunda traza y nombrada aquí "Traza SE", y que aparenta mostrar las evidencias morfológicas de mayor actividad, se ubica a unos $150 \mathrm{~m}$ al sur de la anterior, la cual cruza el abanico de Quigua en una posición más distal. Esta traza se expresa por un escarpe suavizado con exposiciones contrarias, resultantes del movimiento dextral en $60 \mathrm{~m}$ de la topografia abombada característica de un abanico aluvial, indistinto de su granulometría (FIGURA 11). En el sector noroccidental del abanico, la interacción cinemática de la FB con el abanico genera un contraescarpe de exposición $\mathrm{NO}$, de hasta $1,5 \mathrm{~m}$ de altura, que funciona como lomo de obturación (FIGURA
4, entre las 2 flechas más cercanas), mientras más al este se expresa como un escarpe de poca magnitud y exposición SSE, en el mismo sentido que la pendiente topográfica del abanico (FIGURA 12a-c), sobre el que se excavó la trinchera de forma perpendicular al rumbo local de la falla (FIGURAS 11 y 12b). La trinchera, de una profundidad promedio de $2 \mathrm{~m}$, anchura promedio de $1,50 \mathrm{~m}$ y longitud de $9 \mathrm{~m}$, fue ubicada y excavada ortogonal al escarpe suavizado de exposición SSE de la FB, preservado en el tope del abánico de escombros de edad pleistocena (FIGURAS 11 y 12). A pesar de conocerse previo a la ejecución de la excavación, que este abanico resultaba de la depositación de flujos de escombros de origen fluvio-torrencial, por la presencia de bloques métricos protuberantes en su superficie, siempre se contó identificar los eventos sísmicos a partir del criterio de cuña coluvial (ver Audemard, 2005). 

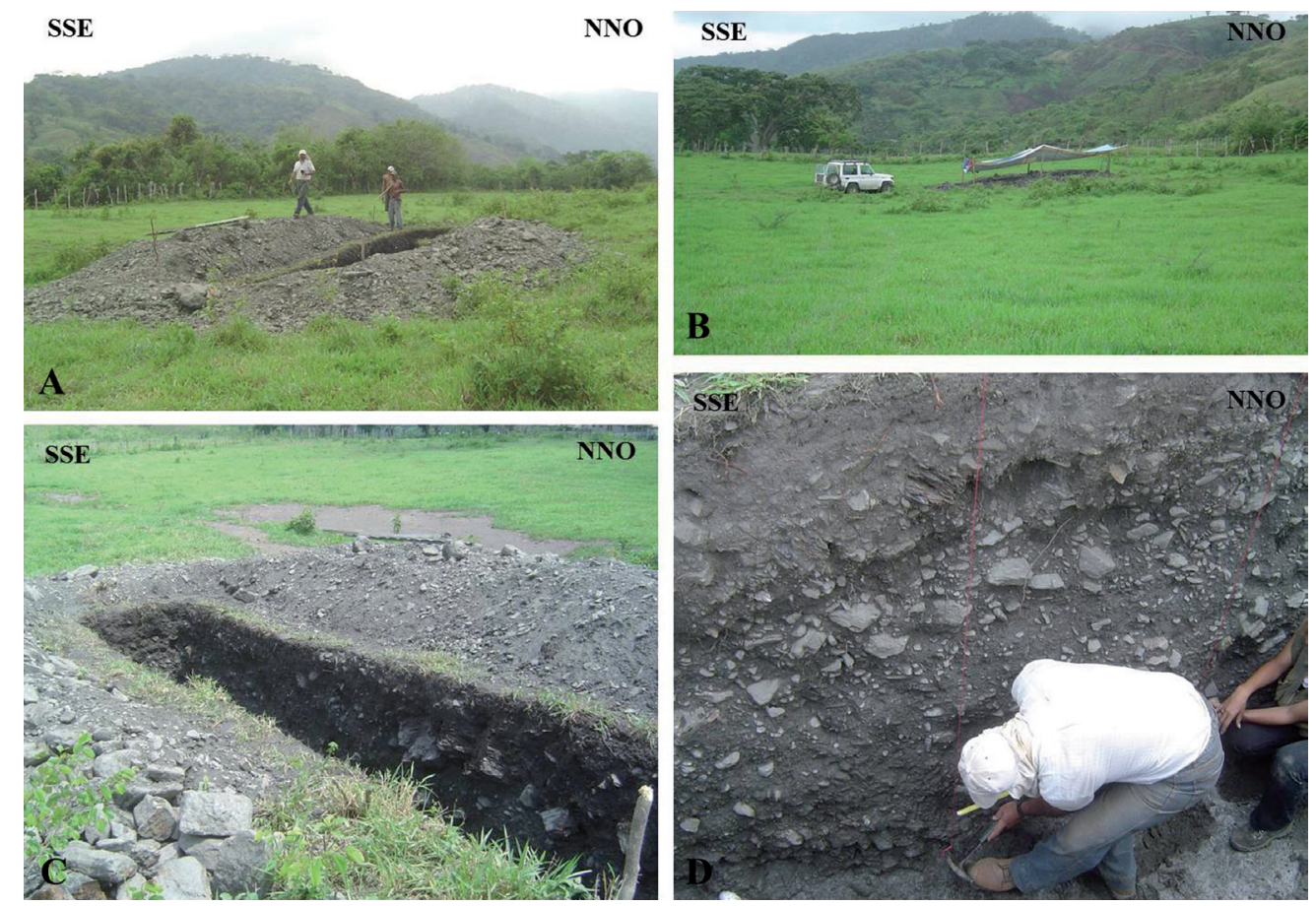

FIGURA 12. Sitio de excavación de la trinchera paleosísmica de Quigua. A) Vista hacia el NO del sitio de excavación, en referencia con la sierra de Aroa al fondo. B) Vista hacia el OSO, longitudinal a la FB, donde se puede apreciar el desnivel introducido por la falla (bloque SSE deprimido). A tal fin, comparar los neumáticos del vehículo y el pasto que circunda los escombros removidos; o usar la posición altitudinal de los topes de los horcones de madera de las cercas de alambre de pua, en posición más distante. C) Se puede apreciar el desnivel topográfico del terreno a lo largo del tope de la pared oeste de la excavación. Igualmente, nótese el cambio granulométrico de fragmentos más pequeños hacia el SSE y más grandes hacia el NNO. D) A pesar de la granulometría muy gruesa del material que compone el abanico de Quigua, se puede reconocer cierta estratificación, aunque no fuertemente desarrollada.

En esta trinchera se identificó y describió una secuencia de conglomerados, aparentemente caóticos a primera vista (FIGURA 12c), pero con superposición de eventos de origen fluvio-torrencial débilmente estratificados (FIGURA 12d). Sin embargo, estos depósitos también presentan diferencias entre ambos extremos de la excavación (SSE y NNO), realzando la presencia de la falla. Hacia el extremo SSE se observa una secuencia de conglomerados con dominio de matriz arcillosa, mientras, en la sección noroccidental dominan bloques de mayor dimensión, los cuales pueden alcanzar el metro de diámetro (FIGURA 12c). Por otra parte, este tipo de bloques en algunas ocasiones controla la sedimentación. La zona de deformación frágil está dominada por fallas paralelas y sub-paralelas que se proyectan en la vertical de las paredes de la trinchera, afectando gran parte de la secuencia sedimentaria expuesta por la excavación (FIGURA 13). Estas fallas coinciden con el escarpe sísmico de exposición SSE, que aparentan estar selladas por las unidades estratigráficas más recientes, que terminan por suavizar el escarpe tectónico (FIGURA 13).
Identificación de los últimos eventos sísmicos: a pesar de la granulometría muy gruesa de los depósitos expuestos en el perfil de la trinchera, se lograron identificar líneas de cantos y paquetes imbricados de conglomerados, con conspicua geometría de cuña coluvial, así como una grieta abierta rellena, que evidencian la actividad de esta traza de la FB en Quigua (FIGURAS 13 y 14). En función de lo antes descrito, se recolectaron un total de 15 muestras de material orgánico, para así realizar dataciones radiocarbónicas (TABLA 2 y FIGURAS 13 y 14), a fin de acotar el momento de ocurrencia de los principales eventos sísmicos.

El evento más antiguo registrado en esta excavación lo soporta la muestra VEN-58-05, con una edad no calibrada de 20660-20860 a AP, el cual no pudo ser calibrado, ya que al momento de realizar los análisis el laboratorio no poseía curvas de calibración para muestras con edad mayor a 20.000 años. Está registrado por una grieta abierta en forma de embudo, en la terminación superior de uno de los planos de falla (FIGURA 14; evento I), la cual está rellena de material coluvial en una franja de deformación que alcanza $40 \mathrm{~cm}$ de ancho en el tope del embudo. 


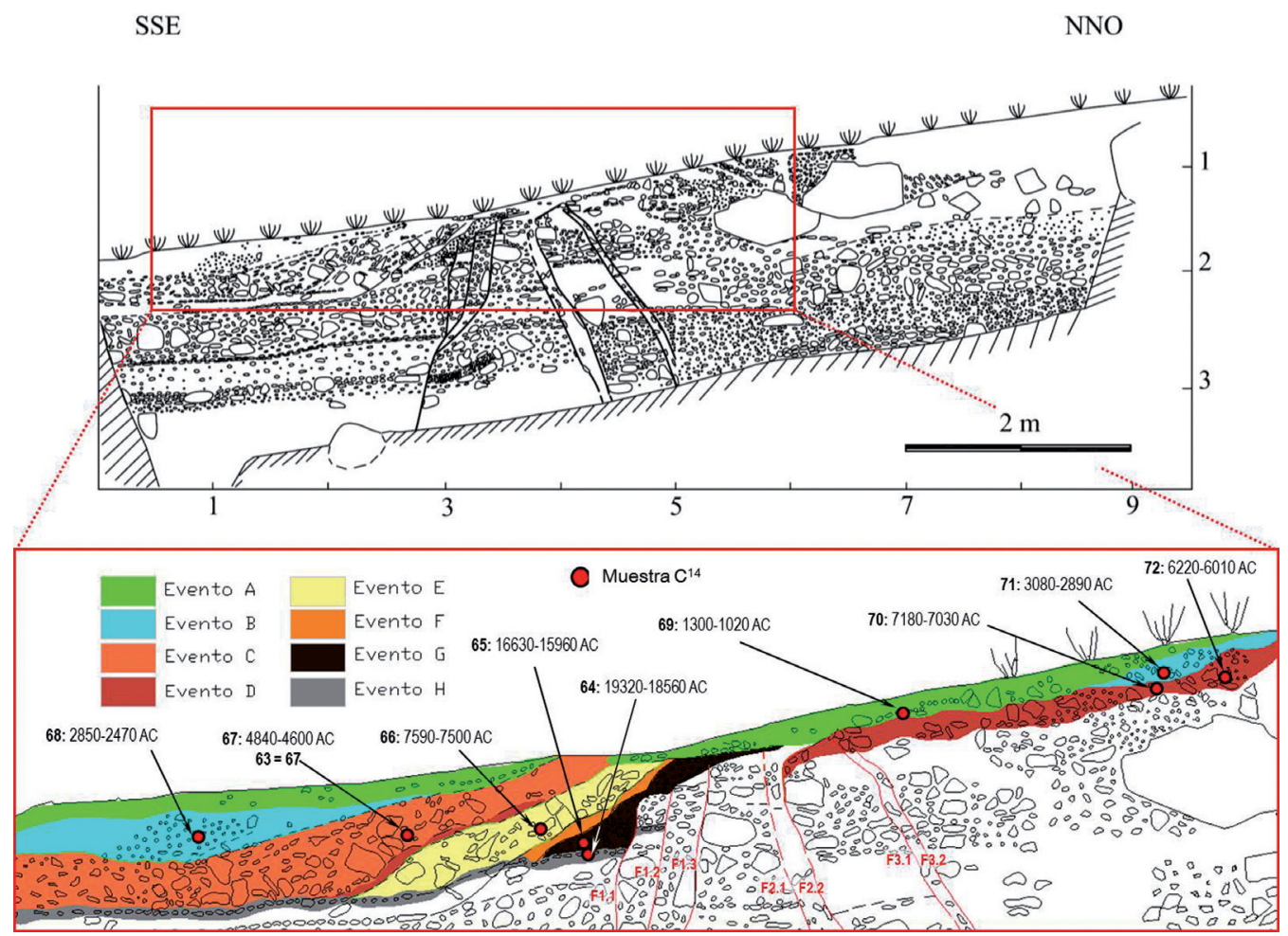

FIGURA 13. Levantamiento geológico de la pared oeste de la trinchera Quigua (-T9- en la FIGURA2). Se reportan todos los puntos de muestreo para datación radiocarbónica, así como sus edades calendario calibradas con 2 sigma de desviación. La ocurrencia de los sismos está aquí evidenciada por la formación de cuñas coluviales derivadas de la erosión del escarpe de falla (ver Audemard, 2005). Es importante recalcar que pareciese que el coluvión más reciente identificado no sella efectivamente las fallas F2.1 y F2.2.

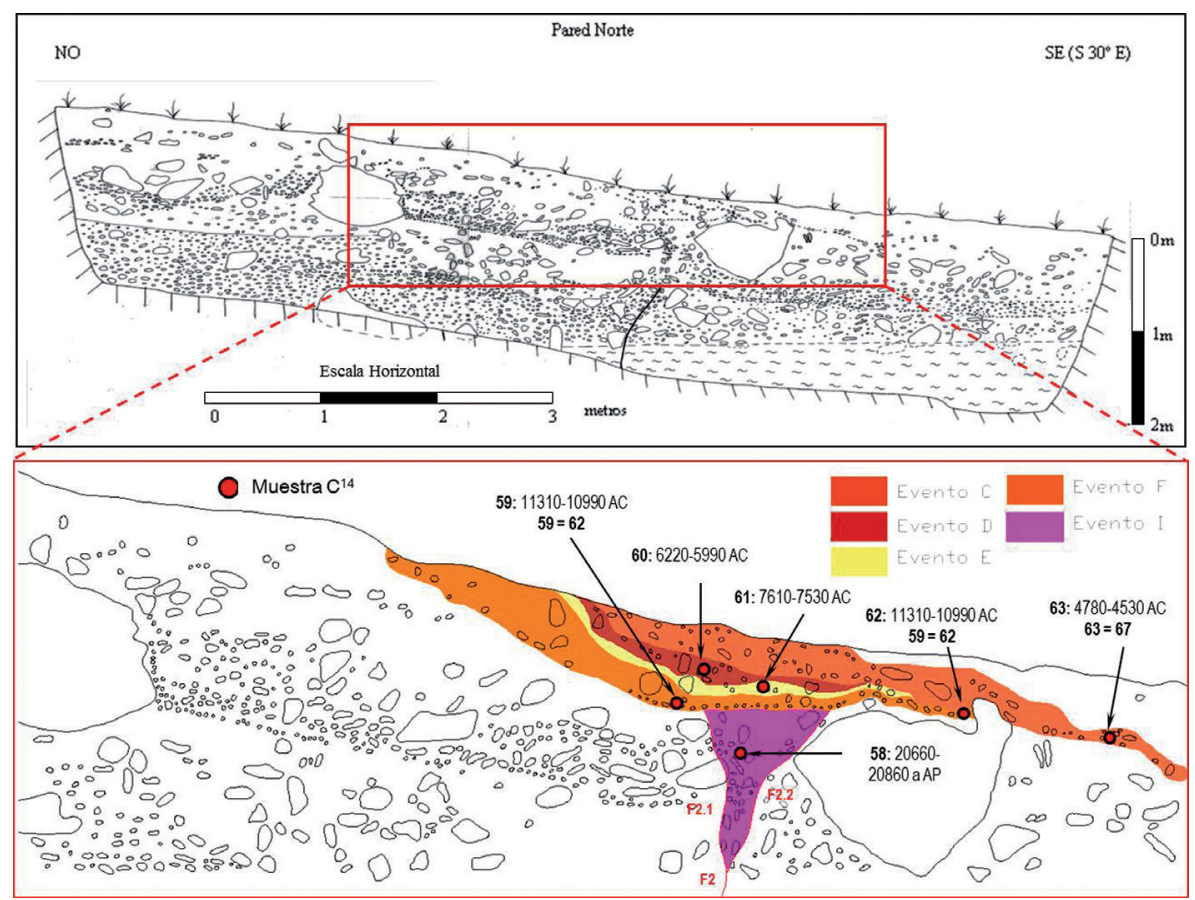

FIGURA 14. Levantamiento geológico la pared este de la trinchera Quigua (-T9- en la FIGURA 2; dibujo del levantamiento por Reinaldo Ollarves). Se reportan todos los puntos de muestreo para datación radiocarbónica, así como sus edades calendario calibradas con 2 sigma de desviación. La ocurrencia de los sismos está aquí evidenciada por la formación de cuñas coluviales derivadas de la erosión del escarpe de falla y en una ocasión, por una grieta abierta rellena subyacente (ver Audemard, 2005). 
TABLA 2. Dataciones radiocarbónicas de muestras colectadas en la trinchera Quigua (T9). la ubicación de los sitios de muestreo está señalado en las FIGURAS 13 y 14.

\begin{tabular}{|c|c|c|c|c|}
\hline \multirow{2}{*}{$\begin{array}{c}\mathrm{N}^{\circ} \\
\text { MUESTRA } \\
\text { FUNVISIS }\end{array}$} & \multirow{2}{*}{$\begin{array}{c}\mathrm{N}^{\circ} \\
\text { BETA } \\
\text { ANALYTIC }\end{array}$} & \multirow{2}{*}{$\begin{array}{c}\text { AÑOS AP CALIBRADOS } \\
2 \text { sigma } \\
\text { (años) }\end{array}$} & \multicolumn{2}{|c|}{$\begin{array}{c}\text { AÑN CALENDARIO } \\
\text { CALIBRADOS }\end{array}$} \\
\hline & & & 1 sigma & 2 sigma \\
\hline VEN-58-05 & 221873 & $20.660-20.860 \#$ & Sin calibración posible & \\
\hline VEN-59-05 & 221874 & $13.260-12.940$ & $11.260-11.100 \mathrm{AC}$ & $11.310-10.990 \mathrm{AC}$ \\
\hline VEN-60-05 & 221875 & $8.170-7.940$ & $6.100-6.020 \mathrm{AC}$ & $6.220-5.990 \mathrm{AC}$ \\
\hline VEN-61-05 & 221876 & $9.560-9.480$ & 7.600-7.570 AC & $7.610-7.530 \mathrm{AC}$ \\
\hline VEN-62-05 & 221877 & $13.260-12.940$ & $11.260-11.100 \mathrm{AC}$ & $11.310-10.990 \mathrm{AC}$ \\
\hline VEN-63-05 & 221878 & $6.730-6.480$ & $4.720-4.590 \mathrm{AC}$ & $4.780-4.530 \mathrm{AC}$ \\
\hline VEN-64-05 & 221879 & $21.280-20.510$ & $19.210-18.640 \mathrm{AC}$ & $19.320-18.560 \mathrm{AC}$ \\
\hline VEN-65-05 & 221880 & $18.580-17.910$ & $16.580-16.040 \mathrm{AC}$ & $16.630-15.960 \mathrm{AC}$ \\
\hline VEN-66-05 & 221881 & $9.540-9.450$ & 7.580-7.530 AC & $7.590-7.500 \mathrm{AC}$ \\
\hline VEN-67-05 & 221882 & $6.790-6.550$ & $4.790-4.690 \mathrm{AC}$ & $4.840-4.600 \mathrm{AC}$ \\
\hline VEN-68-05 & 221883 & $\begin{array}{l}4.800-4.760 \\
4.690-4.680 \\
4.640-4.420\end{array}$ & $2.620-2.480 \mathrm{AC}$ & $\begin{array}{l}2.850-2.810 \mathrm{AC} \\
2.740-2.730 \mathrm{AC} \\
2.690-2.470 \mathrm{AC}\end{array}$ \\
\hline VEN-69-05 & 221884 & $3.250-2.980$ & $1.260-1.120 \mathrm{AC}$ & $1.300-1.020 \mathrm{AC}$ \\
\hline VEN-70-05 & 221885 & $9.120-8.980$ & $7.080-7.050 \mathrm{AC}$ & $7.180-7.030 \mathrm{AC}$ \\
\hline VEN-71-05 & 221886 & $\begin{array}{l}5.030-5.010 \\
4.980-4.840 \\
\end{array}$ & $\begin{array}{l}3.010-2.970 \mathrm{AC} \\
2.960-2.900 \mathrm{AC}\end{array}$ & $\begin{array}{l}3.080-3.060 \mathrm{AC} \\
3.030-2.890 \mathrm{AC}\end{array}$ \\
\hline VEN-72-05 & 221887 & $8.170-7.960$ & $\begin{array}{l}6.200-6.140 \mathrm{AC} \\
6.110-6.050 \mathrm{AC}\end{array}$ & $6.220-6.010 \mathrm{AC}$ \\
\hline
\end{tabular}

\# Edad convencional (años AP)

Los restantes eventos sísmicos se interpretan a partir de cuñas coluviales, cuyas edades con 2 sigma de incertidumbre (TABLA 2 y FIGURAS 13 y 14) están acotadas en los rangos: 19320-18560 AC (VEN-6405), 16630-15960 AC (VEN-65-05), 11310-10990 AC (VEN-59 Y -62-05), 7610-7500 AC (VEN-61 y -66-05), 6220-5990 AC (VEN-60-05), 4840-4530 AC (VEN-63 y -67-05), 2850-2470 AC (VEN-68-05) y eventualmente un último en 1300-1020 AC.

Cabe señalar que esta interpretación no es única porque: a) la cuña coluvial que ha sido individualizada en la pared este con edad de 6220-5990 AC (VEN-60-05; FIGURA 14), parece ser un único cuerpo en la pared contraria con un rango de edad que se extiende entre 7180 y 6010 AC (VEN-70 y 72-05; FIGURA 13); b) el último coluvión, datado por la muestra VEN-69-05, no sella convincentemente los planos de falla intermedios (F2.1 y F2.2 en FIGURA 13). Más aún, al mirar con cuidado, la línea de cantos que define su base está desplazada verticalmente en una decena de centímetros, lo cual permite interpretar que un sismo aún más reciente haya podido ocurrir. Aunque el salto aparenta ser sólo de unos 10 centímetros, no debemos olvidar que la FB es principalmente transcurrente dextral, y que drenajes menores en el tope actual del abanico registran movimientos dextrales recientes de hasta $7 \mathrm{~m}$ (FIGURAS 5 y 11); c) a consecuencia de las difíciles condiciones de afloramiento de las paredes de la trinchera, por lo grosero de la granulometría expuesta, no se puede excluir que algunos eventos no hayan sido develados durante este estudio; y d) dado que las cuñas coluviales requieren de escorrentía natural para formarse luego de cada sismo, no se puede en este caso asegurar que el registro sedimentario en esta localidad sea continuo y esté completo, dado lo intermitente y eventualmente catastrófico del funcionamiento de los flujos de escombros que generaron el abanico fluvio-torrencial de Quigua, que hoy día claramente está aislado a la margen derecha del río que lo ha estado alimentando.

De lo antes expuesto, se interpreta que el periodo de retorno en la FB en esta localidad de Quigua no es regular, asumiendo que hayamos determinado todos los sismos realmente ocurridos sobre esta falla, así como que todos los coluviones determinados sean de origen tectónico y no climático; aún a pesar de descartar el evento más antiguo (aquel identificado por la cuña abierta rellena) que no podemos comparar por estar su edad expresada en otro marco referencial. Los valores de retorno oscilan 
entre 1.500 y 5.100 años. En el supuesto que el último evento no hubiese sido identificado, a pesar de estar la evidencia expuesta en la trinchera (línea de canto basal desplazada del coluvión más reciente observado en la pared oeste), y que el mismo correspondiese al sismo del 26 de marzo de 1812, convincentemente evidenciado en la trinchera de Yaritagua, el retorno entre esos dos últimos sismos sería de unos 3.000 años. Por otra parte, el valor modal del periodo de retorno de la secuencia de sismos establecida oscila entre 2.500 y 3.000 años. Por último, si consideramos que la falla ha sufrido nueve eventos sísmicos en los últimos 21.000 años, lo cual incluiría un sismo más reciente que 1300-1020 AC, y que todos ellos fuesen iguales -lo cual no es claramente el caso-, la FB en el segmento Boc-e recurriría cada 2.300 años aproximadamente.

Asumiendo al menos sismos de magnitud $\mathrm{M}_{\mathrm{s}}$ 7,0 por tener ruptura superficial sobre una falla transcurrente tal como la FB, cuyo desplazamiento cosísmico se aproxima a $1 \mathrm{~m}$, tras aplicar las relaciones de Wells and Coppersmith (1994), la tasa de desplazamiento del segmento Boc-e sería claramente inferior al $1 \mathrm{~mm} / \mathrm{a}$. Si consideramos que el sismo del 26 de marzo de 1812 es representativo de los sismos destructores sobre este segmento Boc-e, cuya magnitud fue estimada en $M_{w i}$ $7,40 \pm 0,35$ por Choy et al. (2010), su desplazamiento cosísmico aplicando las mismas relaciones, estaría entre 1,15 y $4,5 \mathrm{~m}$, pero requiriendo en el caso de mayor magnitud $\left(\mathrm{M}_{\mathrm{wi}} 7,75\right)$ una ruptura de $350 \mathrm{~km}$ de largo, la cual parece altísimamente improbable. Por el contrario, el valor promedio de magnitud obtenido por Choy et al. (2010) requeriría una ruptura de un segmento de unos $140 \mathrm{~km}$ de largo (valor más ajustado a las dimensiones del segmento Boc-e), cuya magnitud resultaría de un salto cosísmico de unos 2,5 m de desplazamiento. En tal caso, la tasa de movimiento se aproximaría al $1 \mathrm{~mm} / \mathrm{a}$ para este segmento Boc-e de la FB. Todo el análisis antes expuesto es únicamente válido en el caso que la traza excavada sea única. Antes hemos expuesto que esta traza aparenta concentrar los mejores y más conspicuos criterios geomórficos de actividad tectónica cuaternaria, pero no es la única traza en la localidad estudiada, pudiendo estar la actividad distribuida en más de una traza activa; en cuyo caso está evaluación no captaría la totalidad de la actividad del segmento Boc-e.

\section{DISCUSIÓN Y CONCLUSIONES}

Más allá de las evidencias geomorfológicas, reportadas por diversos autores (Schubert, 1980; 1982; 1983; Casas, 1991; 1995; Bueno et al., 2005a; Bueno, 2009; entre otros), de fallamiento cuaternario muy claras, frecuentes, consistentes y numerosas que por una parte, identifican la extensión y ubicación de la traza de la FB en su segmento Boc-e, y por otra, determinan su actividad cuaternaria, las 2 excavaciones paleosísmicas ejecutadas sobre este segmento Boc-e, aquí presentadas y discutidas, confirman no sólo su actividad cuaternaria, sino la actividad holocena reiterada en la localidad de Quigua y hasta histórica en la trinchera de Yaritagua.

Ambas trincheras, cuyas interpretaciones paleosísimcias a priori se preveían que reposarían en los criterios de cuña coluvial y/o grieta abierta rellena (referirse a Audemard, 2005, para más detalles), no dejaron de generar sorpresas; y con ello, enseñanza. Por una parte, la trinchera de Yaritagua, a pesar de presentar una estratificación bastante contrastada y fina, tanto en granulometría como en espesor de capas, y materiales datables con buena representatividad en la secuencia, sólo permite identificar el último sismo (el más reciente), que resultó ser histórico y más joven que 1770 DC; así como datar los canales aluviales preservados en el fondo de la trinchera de falla (al menos la sección superior expuesta en la excavación) en menos de 500 años (relleno aluvial igualmente histórico para Venezuela, cuya conquista ocurre durante el tercer viaje de Cristóbal Colón en 1498 DC). Ello resulta de que la gran mayoría (14 de 18) de las muestras tomadas para datación por radiocarbono arrojaron ser modernas (>1950 DC). En consecuencia, los nueve niveles o líneas de cantos identificados y reconocidos dentro del coluvión de ladera durante la fase de excavación (Ollarves et al., 2005), que tapiza el escarpe de falla, deben reinterpretarse como eventos climáticos ocurridos muy recientemente. En tal caso, estas precipitaciones fuertes registradas por las líneas de cantos nos señalan que estos eventos climáticos tienen períodos de retorno próximos a los 50 años (9 eventos en menos de 500 años). No se descarta que las dataciones hayan sido "contaminadas" de manera natural; o eventualmente rejuvenecidas por sucesivas fases de "tala y quema" practicadas por los agricultores locales con fines agrícolas (contaminación antrópica); práctica muy frecuente en la Venezuela rural, como en otros países latinoamericanos y del tercer mundo. De igual manera, podemos rescatar que las dataciones ${ }^{14} \mathrm{C}$ obtenidas de muestras colectadas dentro del canal a más de $1 \mathrm{~m}$ de profundidad sí arrojaron valores distintos a "moderno", siendo ambas dataciones congruentes y próximas a 500 años de antigüedad. Es de recalcar que las 2 muestras corresponden a materiales distintos: mientras una está representada por carbón vegetal, la otra proviene de un suelo rico en materia orgánica. Por otra parte, a sabiendas que la trinchera de Quigua daría dificultades por su granulometría grosera de flujo de escombro, por estar cortada a expensas de un abanico de generación episódica fluvio-torrencial, no se esperaba que los sismos mayores (superiores a $M_{w} 7,0$ ) de la FB recurrieran según 
una moda entre 2.500 y 3.000 años, y con una variación tan dispersa como entre 1.500 y 5.100 años; o de manera promediada cada 2.300 años (unos 9 sismos en una ventana temporal de 21.000 años). Es bien sabido que en cualquier evaluación paleosísmica cabe la posibilidad de subinterpretación y sobreinterpretación de la cronología de los eventos, y en el caso particular de la trinchera de Quigua, la naturaleza de los procesos de construcción de este abanico fluvio-torrencial introduce mayor incertidumbre en la identificación e interpretación de los eventos sismicos con base en criterios sedimentarios. Aún considerando que aquí se hayan conjurado todos los factores negativos posibles de orden sedimentario: a) registro incompleto porerosión introducida porun episodio fluvio-torrencial de gran energía, b) carencia de registro sedimentario porque el abánico queda desconectado de su río y no recibe más sedimentos -en posición más elevada que los posteriores y más recientes episodios de crecida; que parece ser el caso-, c) pobre desarrollo de estratificación que dificulte el reconocimiento de los episodios sedimentarios, así como la formación de cuñas coluviales y grietas abiertas rellenas, y d) escorrentía superficial de poco poder de transporte, sin capacidad de rectificar el escarpe tectónico y generar las subsecuentes cuñas coluviales, es necesario contemplar entonces dos escenarios posibles: 1) la FB es necesariamente mucho más lenta en este segmento Boc-e que sus equivalentes andinos (Boc-a a Boc-d; ver Audemard and Audemard, 2002) con una tasa de movimiento entre 1 y $3 \mathrm{~mm} / \mathrm{a}$ para este segmento Boc-e, tal como postula Casas (1991; 1995). El valor mínimo propuesto por este autor sería consistente con que la falla se mueva con $\operatorname{sismos} \mathrm{M}_{\mathrm{w}} 7,4$ -equivalente a la magnitud del sismo de 1812 estimada por Choy et al. (2010) o la propuesta por Audemard et al. (2000)-, producto de un desplazamiento cosísmico de 2,5 m según las relaciones de Wells and Coppersmith (1994) para fallas transcurrentes, con un tiempo de recurrencia de 2.300 años (valor promedio derivado de considerar la ocurrencia de nueve eventos en 21.000 años de historia paleosísmica); o 2) otra(s) traza(s) subparalela(s) a la ubicada en la Hacienda El Playón, aquella cortando el abanico de Quigua y aquí explorada, distribuye(n) la deformación. Éste podría ser el caso, dado que se ha identificado al menos otra traza (FIGURA 11), la cual está adosada al pie del relieve. Bueno (2009) considera ésta la traza principal, y así justifica el largo periodo de recurrencia entre sismos determinado en la excavación de Quigua.

En términos del último sismo interpretado en esta evaluación paleosísmica, los resultados arrojados por la trinchera de Yaritagua determinan que la más reciente ruptura superficial cumple con las siguientes condiciones: a) está asociada a la $\mathrm{FB}$, dado que la trinchera explora esta falla; b) presenta una magnitud próxima a $\mathrm{M}_{\mathrm{w}} 7,0$ o superior, para lograr romper la superficie del terreno (por intermedio de grietas abiertas, en este caso), durante un sismo transcurrente; y c) ocurrió después de 1770 DC, según datación radiocarbónica de la parte profunda de la grieta abierta rellena que evidencia este evento (FIGURA 10). Por su parte, el coluvión más reciente expuesto por la trinchera de Quigua, no aparenta convincentemente sellar la zona de falla subyacente, pudiendo implicar que sí ocurrió una reactivación post-1000 años AC (FIGURA 13); y probablemente tuvo lugar en tiempos históricos, si aceptamos que la moda del tiempo de recurrencia de la FB está entre 2.500 y 3.000 años, al menos en la traza explorada. Una línea de cantos intra-último coluvión parece soportar lo antes expresado, ya que exhibe un salto vertical del orden de unos $10 \mathrm{~cm}$, en la proyección vertical hacia arriba de unos de los planos de falla en posición intermedia de la zona de deformación frágil (FIGURA 13).

Comparando los resultados antes mencionados con los sismos históricos conocidos para esta región del país (Centeno Graü, 1940; 1969; Grases, 1990; Rodríguez y Audemard, 1997; Grases et al., 1999; Altez, 2005a; 2006; Choy et al., 2010), el único evento sísmico en los últimos 250 años con daños ocasionados en el valle del Yaracuy y con una magnitud, estimada a partir de esos daños por diversos autores (Fiedler, 1972; Grases, 1990; Grases y Rodríguez, 2001; Choy et al., 2010), al menos igual a 7,0 o superior, es el sismo del 26 de marzo de 1812. Así queda corroborado por vía geológica que el terremoto de 1812 que destruye Barquisimeto y San Felipe (en el valle del Yaracuy), al igual que Caracas, es atribuible al segmento Boc-e de la Falla de Boconó, al menos en su extensión adosada a la sierra de Aroa. Probablemente también incorpora el tramo más septentrional de Albarico-golfo Triste del segmento Boc-e, si su magnitud ciertamente se aproxima a $\mathrm{M}_{\mathrm{w}} 7,4$, tal como la ha estimado Choy et al. (2010) para el sismo de 1812 y la ha propuesto Audemard et al. (2000) para todo el segmento Boc-e de la Falla de Boconó. La longitud de la ruptura concordaría bastante bien con la isosista VIII propuesta para el subevento del valle del Yaracuy por Rodríguez y Audemard (1997) y Altez (2005a; 2006).

\section{AGRADECIMIENTOS}

Quiero agradecer a los colegas del Departamento Ciencias de la Tierra, Ings. Gustavo Díaz, Luz María Rodríguez y Reinaldo Ollarves, por su aporte durante el reconocimiento de campo que sirvió como base para la identificación morfotectónica del segmento San Felipe 
de la Falla de Boconó, que posteriormente conllevara a la ubicación de las excavaciones paleosísmicas aquí ejecutadas. Al Ing. Ollarves reitero tal agradecimiento por las horas igualmente compartidas durante el posterior estudio paleosísmico. Cabe también mencionar el apoyo de la Ing. Mary Luz Bueno, tanto en la fase de cartografía como de evaluación paleosísmica, quien se encontraba durante dichos estudios en fase de entrenamiento; actividad enmarcada dentro de su tesis de maestría (aún inconclusa). Al siempre presto profesor y amigo André Singer, por su disposición a la discusión fructífera. Merecen igualmente mi gratitud la cuadrilla de arduos excavadores. La siempre presta, eficiente y excelente dibujadora Marina Peña González, por la elaboración de la mayoría de las figuras. Las edades radiocarbónicas fueron todas obtenidas en el laboratorio norteamericano Beta Analytic Inc. (Miami, Florida). Esta investigación es una contribución a los Proyectos FONACIT 2001002492 (Agenda Gestión de Riesgos y Manejo de Desastres), el cual proveyó los fondos, FONACIT 2002000478 (GEODINOS), y los Proyectos FONACIT-ECOS Nord PI-2003000090 y PI-2009000818. Queremos expresar particular reconocimiento al Dr. Raúl Estevez, profesor jubilado de la ULA, gestor de la Agenda Gestión de Riesgos y Manejo de Desastres del FONACIT, a comienzos de este siglo XXI. Se quiere igualmente dejar constancia de mi agradecimiento a los tres revisores, Drs. Andrés Folguera, Victor Ramos y Guillermo Sagripanti por sus sugerencias; todos colegas tectonistas argentinos.

\section{REFERENCIAS}

Aggarwal, Y. 1983. Seismic gaps and earthquake hazard in Venezuela. Proceedings of Simposio Neotectonica, Sisimicidad y Riesgo Geológico en Venezuela y el Caribe, Caracas, pp. 26.

Altez, R. 1998. Cronometrización extemporánea: los sismos del 26 de marzo de 1812 en Caracas y Mérida, Revista Geográfica Venezolana, 39 (1-2): 297-325.

Altez, R. 2005a. Los sismos del 26 de marzo de 1812 en venezuela: Nuevos aportes y evidencias sobre estos eventos. Boletín Técnico IMME, 43 (2): 11-34.

Altez, R. 2005b. Todo lo que se movió en 1812 en la placa del Caribe: sismos, volcanes y transmisión de energía. Revista Geográfica Venezolana, Número especial 2005: 143-170.

Altez, R. 2006. El Desastre de 1812 en Venezuela: Sismos, Vulnerabilidades y Una Patria No Tan Boba.
Universidad Católica Andrés Bello y Fundación Polar, Caracas, Venezuela, 522p.

Alvarado, M. 2008. Caracterización Neotectónica de la cuenca La González. Estado Mérida, Venezuela. Trabajo Final de Grado para optar al título de Magister Scientiarum, mención Ciencias Geológicas, Postgrado en Ciencias Geológicas, Universidad Central de Venezuela, 89p.

Alvarado, M., Audemard, F.A., Laffaille, J., Ollarves, R., y Rodríguez, L.M., 2007. Análisis paleosismológico de la Falla de Boconó en el sector Lagunillas, Estado Mérida, Venezuela. IX Congreso Geológico Venezolano, Caracas, pp. 7 (formato CD).

Alvarado, M., Audemard, F.A., Laffaille, J., Ollarves, R., and Rodríguez, L.M. 2008. Paleoseismic investigation on the Boconó fault, between Las González and Estanques, Mérida Andes, Venezuela. VII International Symposium on Andean Geodynamics -7 ISAG(resumen extendido).

Alvarado, M., Audemard, F.A., Laffaille, J., Ollarves, R., and Rodríguez, L.M. 2009. Paleoseismic analysis of the Boconó fault, between the La Gonzalez-Estanques towns, Mérida state, Venezuela. VII International Conference on Geomorphology, Melbourne, Australia, 6-11/07/2009.

Audemard, F.A. 1997. Holocene and historical earthquakes on the Boconó fault system, southern Venezuelan Andes: trench confirmation. Journal of Geodynamics 24(1-4): 155-167.

Audemard,F.A. 1998. Contribucióndelapaleosismología a la sismicidad hístorica: los terremotos de 1610 y de 1894 en los Andes venezolanos meridionales. In: Ferrer, C, Laffaille, J. y Rengifo, M. (eds.), Selección de artículos de las Primeras Jornadas de Sismicidad Histórica en Venezuela, Núcleo Universitario Rafael Rangel, ULA-Trujillo, mayo 1997. Revista Geográfica Venezolana, Universidad de Los Andes, Venezuela, 39(1-2): 87-105.

Audemard, F.A. 1999. Morpho-structural expression of active thrust fault systems in the humid tropical foothills of Colombia and Venezuela. In: Frisch, W. (ed.), Proceedings of the Fourth International Conference on Geomorphology Bologna, 1997, Vol. III. Zeitschrift für Geomorphologie, 118: 1-18.

Audemard, F.A. 2001. Historia sísmica y segmentación sismogenética de la Falla de Boconó con base en el análisis 
geológico de sedimentos recientes deformados (por vía de trincheras y núcleos continuos). Propuesta de proyecto introducida ante el FONACIT, 19p (formato digital).

Audemard, F.A. 2002. Ruptura de los grandes sismos históricos venezolanos de los siglos XIX y XX revelados por la sismicidad instrumental contemporánea. XI Congreso Venezolano de Geofísica, pp. 8 (formato CD).

Audemard, F.A. 2003a. Estudios paleosísmicos por trincheras en Venezuela: métodos, alcances, aplicaciones, limitaciones y perspectivas. Revista Geográfica Venezolana, Universidad de Los Andes, 44(1): 11-46.

Audemard, F.A. 2003b. Geomorphic and geologic evidence of ongoing uplift and deformation in the Mérida Andes, Venezuela. Quaternary International 101-102C: 43-65.

Audemard, F.A. 2005. Paleoseismology in Venezuela: objectives, methods, applications, limitations and perspectives. Tectonophysics, 408 (1-4): 29-61.

Audemard, F.A. 2006. Surface rupture of the Cariaco July 09, 1997 Earthquake on the El Pilar fault, northeastern Venezuela. Tectonophysics, 424 (1-2): 19-39.

Audemard, F.A. 2008. Historia sísmica y segmentación sismogenética de la Falla de Boconó con base en el análisis geológico de sedimentos recientes deformados (por vía de trincheras y núcleos continuos). Proyecto Fonacit 2001002492. Informe interno FUN-025, 2008. $83 p+1$ apéndice

Audemard, F.A. 2009a. Falla de Boconó, Venezuela (VE-06b y VE-06c). En Proyecto Mutinacional Andino. Geociencia para las comunidades andinas (eds.): Atlas de deformaciones cuaternarias de Los Andes. Publicación Geológica Multinacional, 7: 260-272.

Audemard, F.A. 2009b. Key issues on the postMesozoic southern Caribbean plate boundary. In: James, K.H., Lorente, M.A. and Pindell, J., (eds): Origin and Evolution of the Caribbean Plate, Geological Society, London, Special Publications, 328: 567-584.

Audemard, F.A. 2009c. Segmentation of the Boconó Fault from paleoseismic trench results, Mérida Andes, Venezuela. VII International Conference on Geomorphology, Melbourne, Australia, 6-11/07/2009 (Abstract; CD Format).

Audemard, F.A. 2010. Seismogenic Fault Segmentation of the Boconó Fault from Paleoseismic Trench Results,
Western Venezuela: NE-Directed Rupture during the Latest Earthquake Cycles? Hokudan International Symposium on Active Faulting 2010, Japan: Forecasting Large Earthquakes from Active Faults in Time and Space: 6-8 (Extended abstract).

Audemard, F.A. 2014. Segmentación sismogenética de la Falla de Boconó a partir de investigaciones paleosísmicas por trincheras, Venezuela occidental: ¿Migración de la ruptura hacia el noreste en tiempos históricos? Revista Asociación Geológica Argentina, 71 (2): 247 - 259.

Audemard, F.A., and Soulas, J.P. 1995. Trench Confirmation of Historical Earthquakes on the Boconó Fault System, Southern Venezuelan Andes. XIV INQUA International Congress -Symposium on Paleoseismology-, Berlín, Alemania (03-10 august); Terra Nostra, 2/95:16.

Audemard, F.A., and Singer, A. 1996. Active Fault Recognition in Northwestern Venezuela and its Seismogenic Characterization: Neotectonic and Paleoseismic approach. In: Enghdal, E.R., Castano, J. \& Berrocal, J. (eds.), Proceedings of the 1994 Regional Seismological Assembly in South America. Geofísica Internacional, México, 35(3): 245-255.

Audemard, F.E., and Audemard, F.A. 2002. Structure of the Mérida Andes, Venezuela: relations with the South America-Caribbean geodynamic interaction. Tectonophysics, 345(1-4): 299-327.

Audemard, F.A., and Michetti, A.M., 2011. Geological criteria for evaluating seismicity revisited: 40 years of paleoseismic investigations and the natural record of past earthquakes. In Audemard, F. A., Michetti A. M. and Mccalpin, J. (eds.) Geological criteria for evaluating seismicity revisited: 40 years of paleoseismic investigations and the natural record of past earthquakes, GSA Special papers $\mathrm{N}^{\circ} 479$, Boulder. 1-21. doi:10.1130/2011.2479(00).

Audemard, F.A., Pantosti, D., Machette, M., Costa, C., Okumura, K., Cowan, H., Diederix, H., Ferrer, C., and Sawop Participants. 1999a. Trench investigation along the Merida section of the Boconó fault (central Venezuelan Andes), Venezuela. In: Pavlides, S., Pantosti, D. and Peizhen, Z. (eds.), Earthquakes, Paleoseismology and Active Tectonics. Selected papers to $29^{\text {th }}$ General Assembly of the Association of Seismology and Physics of the Earth's Interior (IASPEI), Thessaloniki, Greece, August 1997. Tectonophysics, 308: 1-21. 
Audemard, F.A., Romero, G., y Rendón, H. 1999b. Sismicidad, Neotectónica y Campo de Esfuerzos del Norte de Venezuela. FUNVISIS unpublished Report for PDVSA-CVP, 221p.

Audemard, F.A., Machette, M., Cox, J., Dart, R., and Haller, K. 2000. Map and Database of Quaternary Faults in Venezuela and its Offshore Regions. US Geological Survey Open-File Report 00-0018. Comprende mapa a escala 1:2.000.000 e informe, 78p.

Audemard, F.A., Romero, G. Rendón, H., and Cano, V. 2005. Quaternary fault kinematics and stress tensors along the southern Caribbean from microtectonic data and focal mechanism solutions. Earth-Science Reviews, 69 (3-4): 181-233. doi: 10.1016/j. earscirev.2004.08. 001

Audemard, F.A., Carrillo, E., and Beck, C. 2007. Fieldtrip Guidebook for International Workshop on "Blind dip-slip faulting and strain partitioning in an active orogen: The Mérida Andes case, Venezuela", Santo Domingo, estado Mérida, Venezuela, March 0509, 2007, 77p.

Audemard, F.A., Ollarves, R., Betchtold, M., Díaz, G., Beck, C., Carrillo, E., Pantosti, D., and Diederix, H. 2008. Trench investigation on the main strand of the Boconó fault in its central section, at Mesa del Caballo, Mérida Andes, Venezuela. Tectonophysics, 459: 38-53.

Bakun, W.H., and Wentworth, C.M., 1997. Estimating earthquake locations and magnitudes from seismic intensity data, Bulletin of the Seismological Society of America, 87: 1502-1521.

Bakun, W.H., and Wentworth, C.M. 1999. Erratum to estimating earthquake locations and magnitudes from seismic intensity data, Bulletin of the Seismological Society of America, 89: 557.

Beltrán, C. 1994. Trazas activas y síntesis neotectónica de Venezuela a escala 1:2.000.000. Proceedings VII Congreso Venezolano de Geofísica, Caracas, pp. 541-547.

Boinet, T. 1985. La frontière méridionale de la plaque caraïbe aux confins colombo-vénézuéliens (Norte de Santander, Colombie): données géologiques. Ph.D. Thesis, Université de Paris VI, Paris, France, 204p + appendices.

Bueno, M. 2009. Registro paleosísmico en el segmento Cabudare-Morón de la Falla de Boconó cerca de la cuidad de San Felipe, Venezuela. V Congreso Venezolano de
Sismología e Ingeniería Sísmica, Caracas, Venezuela. Pp. 13 (Formato CD).

Bueno, M.L., Audemard, F.A., Ollarves, R., y Rodriguez, L.M. 2005a. Cartografía neotectónica de la Falla de Boconó entre las poblaciones de Boconó y Urama, para fines de identificación de sitios propicios para excavaciones paleosísmicas. FUNVISIS. Informe Inédito, $47 \mathrm{p}$.

Bueno, M.L., Ollarves, R., y Audemard, F. A. 2005 b. Interpretaciones preliminares en la caracterización sismogénica de la Falla de Boconó en el segmento Sabana Grande-Sanare (estado Lara), con base en dos excavaciones paleosísmicas. IV Coloquio sobre Microzonificación Sísmica, Barquisimeto, Nov. 2005. Serie Técnica Funvisis 1-2005, pp. 67-72.

Bull, W. 1977. The alluvial-fan environment. Progress in Physical Geography, 1(2): 222-270.

Bull, W. 1984. Alluvial fans and pediments of southern Arizona. In: Smiley, T.L., Nations, J.D., Pewe T.L., and Schafer J.P. (eds.): Landscapes of Arizona. University Press of America. Chapter 11, pp. 229-252.

Casas, A.M. 1991. Estudio sismotectónico del valle de Yaracuy. Informe inédito, Fundación Venezolana de Investigaciones Sismológicas (FUNVISIS, Caracas), $123 p$.

Casas, A.M. 1995. Geomorphological and sedimentary features along an active right-lateral reverse fault (Yaracuy basin, Venezuela). Zeitschrift für Geomorphologie, 39(3): 363-380.

Centeno Graü, M. 1940. Estudios Sismológicos. Litografía del Comercio, Caracas.

Centeno Graü, M. 1969. Estudios Sismológicos. Academia Nacional de Ciencias Físicas, Matemáticas y Naturales, Talleres Tipo-Litográficos de la Dirección de Cartografía Nacional, Caracas, segunda edición, 366p.

Choy, J. 1998. Profundidad y mecanismo focal del terremoto de El Tocuyo, 1950. Revista Geográfica Venezolana, 39(1-2): 203-217.

Choy, J.E., Palme, C., Guada, C., Morandi, M., y Klarica, S. 2007. Incorporación de intervalos de intensidades y de corrección por rumbo de fallas al método de Bakun \& Wentworth para la evaluación de sismos históricos, IX Congreso Geológico Venezolano, Caracas, Venezuela. 
Choy, J., Palme, C., Guada, C., Morandi, M., and Klarica S. 2010. Macroseismic Interpretation of the 1812 Earthquakes in Venezuela Using Intensity Uncertainties and A Priori Fault-Strike Information. Bulletin of the Seismological Society of America, 100(1): 241-255.

Cluff, L., and Hansen, W. 1969. Seismicity and Seismic Geology of Northwestern Venezuela. Woodward-Clyde y Associates' unpublished report for Shell de Venezuela. Vol I and II.

Fiedler, G. 1961. Áreas afectadas por terremotos en Venezuela. Memoria del III Congreso Geológico Venezolano, 3: 1791-1810. Editorial Sucre, CaracasVenezuela.

Fiedler, G. 1972. La liberación de energía sísmica en Venezuela, volúmenes sísmicos y mapas de Isosistas. Memoria IV Congreso Geológico Venezolano, pp. 2441-2462, Caracas-Venezuela.

Freymueller, J.T., Kellogg, J.N., and Vega, V. 1993. Plate motions in the north Andean region. Journal of Geophysical Research, 98: 21853-21863.

Gasperini, P., Bernardini, F., Valensise, G., and Boschi, E. 1999. Defining seismogenic sources from historical earthquakes felt reports. Bulletin of the Seismological Society of America, 89, 94-110.

Giraldo, C. 1985. Néotectonique et sismotectonique de la région d'El Tocuyo-San Felipe (Vénézuéla CentroOccidental). Tesis PhD Academie de Montpellier. Universite des Sciences et Techniques du Languedoc, 130p.

Grases, J. 1990. Terremotos destructores del Caribe 1502-1990. $1^{\circ}$ ed., Orcyt (UNESCO), Montevideo, Uruguay, $132 \mathrm{p}$.

Grases, J., y Rodríguez, J.A. 2001. Estimaciones de sismos venezolanos a partir de mapas de isosistas. Memorias del 2do Seminario Iberoamericano de Ingeniería Sísmica. Madrid, España.

Grases, J., Altez, R., y Lugo M. 1999. Catálogo de Sismos Sentidos o Destructores Venezuela 1530/1998, Universidad Central de Venezuela/Academia de Ciencias Física, Matemáticas y Naturales, Facultad de Ingeniería, Caracas, Venezuela, 650p.

Guzmán, O., Vassallo, R., Audemard, F.A., Mugnier, J.L., Oropeza, J., Yepez, S., Carcaillet, J., Alvarado, M., and Carrillo, E. 2013. ${ }^{10}$ Be dating of river terraces of Santo Domingo river, on Southeastern flank of the Mérida Andes, Venezuela: tectonic and climatic implications. Journal of South American Earth Sciences, 48: 85-96. doi: 10.1016/j.jsames.2013.09.004

Laffaille, J., y Ferrer, C. 2003. El terremoto del Jueves Santo en Mérida. II Jornadas de Sismología Histórica. Revista Geográfica Venezolana, 44(1):107-123.

Laffaille, J., y Ferrer, C. 2005. El terremoto de Mérida de 1812: escrudiñando entre las páginas de una novela inconclusa en busca de información acerca de una historia real. Revista Geográfica Venezolana, número especial: 217-232.

McCann, W., and Pennington, W. 1990. Seismicity, large earthquakes, and the margin of the Caribbean plate. In Dengo, D. and Case, J.E. (eds.): The Caribbean region. The Geology of North America, v. H., Geological Society of America, pp. 291-306.

Minster, J., and Jordan, T. 1978. Present-day plate motions. Journal of Geophysical Research, 83: 5331-5354.

Molnar, P., and Sykes, L. 1969. Tectonics of the Caribbean and Middle America Regions from focal mechanisms and Seismicity. Geological Society of America Bulletin, 80: 1639-1684.

Ollarves, R., Bueno, M.L., and Audemard, F.A. 2005. Evaluación paleosísmica de los segmentos de falla Yaritagua y Quigua, edo. Yaracuy, en el extremo nororiental de la Falla de Boconó. IV Coloquio sobre Microzonificación Sísmica, Barquisimeto, Nov. 2005. Serie Técnica Funvisis, 1-2005, pp. 183-186.

Ollarves, R., Audemard, F.A., and López, M. 2006. Morphotectonic criteria for the identification of active blind faulting in alluvial environments: case studies from Venezuela and Colombia. In: Latrubesse, E. (ed.) Tropical Geomorphology with special emphasis on South America, Volume VI. Zeitschrift für Geomorphologie, 145: 81-103.

Palme, C., y Altez, R. 2002. Los terremotos de 1673 y 1674 en los Andes venezolanos. Interciencia 27(5): 220-226.

Palme, C., Morandi, M., y Choy, J. 2005a. Reevaluación de las intensidades de los grandes sismos históricos de la región de la cordillera de Mérida utilizando el método de Bakun y Wentworth. Revista Geográfica Venezolana, Número Especial, IV Jornadas Venezolanas de Sismología Histórica y V Simposio Venezolano de Historia de las Geosciencias, número especial, pp.233-253. 
Palme, C., Morandi, M., y Choy, J. 2005b. Determinación de una relación lineal entre intensidad, magnitud y distancia epicentral para el occidente de Venezuela. Interciencia, 30: 195-204.

Palme, C., Altez, R., Silva, J., Morandi, M.T., Rodríguez, J.A., Choy, J., Urbani, F., y Rengifo, M. Sistema de Teleinformación de la Sismología Histórica de Venezuela (STSHV) http://sismicidad.ciens.ula.ve

Pérez, O.J., Bilham, R., Sequera, M., Molina, L., Gavotti, P., Codallo, H., Moncayo, C., Rodríguez, C., Velandia, R., Guzmán M., y Molnar, P. 2011. Campo de Velocidades GPS en el Occidente de Venezuela: Componente lateral derecha asociada a la Falla de Boconó y componente convergente perpendicular a Los Andes. Interciencia, 36(1): 39-44.

Rengifo, M., y Laffaille, J. 1998. El terremoto del año 1894 en los Andes venezolanos. Revista Geográfica Venezolana, 39: 141-162.

Rengifo, M., y Laffaille, J. 2000. Reevaluación del sismo del 28 de abril de 1894. Acta Científica Venezolana, 51: 160-175.

Richter, C.F. 1958. Elementary Seismology. Freeman, San Francisco. 768p.

Rod, E. 1956a. Earthquakes of Venezuela related to strike slip faults? American Association of Petroleum Geologists Bulletin, 40: 2509-2512.

Rod, E. 1956b. Strike-slip faults of northern Venezuela. American Association of Petroleum Geologists Bulletin, 40: 457-476.

Rodríguez, J.A., y Audemard, F.A. (coords.) 1997. Estudio neotectónico y geología de fallas activas en el piedemonte surandino de los Andes Venezolanos (Proyecto INTEVEP 95-061). Informe Inédito para INTEVEP, S.A., $155 p+9$ anexos.

Schubert, C. 1974. Late Pleistocene Mérida Glaciation, Venezuelan Andes. Boreas, 3: 147-152.

Schubert, C. 1980. Morfología neotectónica de una falla rumbo-deslizante e informe preliminar sobre la Falla de Boconó, Andes merideños. Acta Científica Venezolana, 31: $98-111$.

Schubert C. 1982. Neotectonics of the Boconó fault, western Venezuela. Tectonophysics, 85: 205-220.

Schubert C. 1983. La Cuenca de Yaracuy: Una estructura neotectónica en la región centro-occidental de Venezuela. Geología Norandina, 8; 3-11.
Schubert, C., y Krause, F. 1981. Fallamiento a lo largo de la costa norte-central de Venezuela: zona de falla de Morón. II Congreso Venezolano de Sismología e Ingeniería Sísmica. Caracas, pp. 155-184.

Singer, A., and Beltrán, C. 1996. Active faulting in the southern Venezuelan Andes and Colombian borderland. $3^{\text {rd }}$ International Symposium on Andean Geodynamics, Saint-Malo, France, pp. 243-246.

Soulas, J.P. 1985. Neotectónica del flanco occidental de los Andes de Venezuela entre $70^{\circ} 30^{\prime}$ y $71^{\circ} 00^{\prime} \mathrm{W}$ (Fallas de Boconó, Valera, Piñango y del Piedemonte). VI Congreso Geológico Venezolano, vol. 4., pp. 2690-2711.

Soulas, J.P. 1986. Neotectónica y tectónica activa en Venezuela y regiones vecinas. VI Congreso Geológico Venezolano, vol. 10., Caracas, pp. 6961-6999.

Soulas, J.P., Rojas, C., y Schubert, C. 1986. Neotectónica de las fallas de Boconó, Valera, Tuñame y Mene Grande. Excursión No 4. VI Congreso Geológico Venezolano,vol. 10., Caracas, pp. 6961-6999.

Soulas, J.P., Singer, A., y Lugo, M. 1987. Tectónica cuaternaria, características sismogénicas de las fallas de Boconó, San Simón y del piedemonte occidental andino y efectos geológicos asociados a la sismicidad histórica (Proyecto Sumandes). FUNVISIS para MARAVEN, $90 p+$ anexos. Inédito.:

Stephan, J. 1982. Evolution géodynamique du domaine Caraibe Andes et Chaine Caraibe sur la transversale de Barquisimeto (Venezuela). Tesis PhD, Universidad Pierre et Marie Curie, 512p.

Suárez, G., and Nábelek, J. 1990. The 1967 Caracas Earthquake: fault geometry, direction of rupture propagation and seismotectonic implications. Journal of Geophysiscal Research, 95(B11): 17459-17474.

Trenkamp, R., Kellogg, J., Freymueller, J., and Mora, H. 2002. Wide plate margin deformation, southern Central America and northwestern South America, CASA GPS observations. Journal of South American Earth Sciences, 15: 157-171.

Wells, D., and Coppersmith., K. 1994. New empirical relationships among magnitude, rupture length, rupture width, rupture area, and surface displacement. Bulletin of the Seismological Society of America, 84(4): 974-1002.

Trabajo recibido: julio 3 de 2014

Trabajo aceptado: noviembre 25 de 2014 